Olaf Groh-Samberg, Steffen Mau und Uwe Schimank

\title{
Investieren in den Status: Der voraussetzungsvolle Lebensführungsmodus der Mittelschichten ${ }^{1}$
}

\section{Einleitung}

Nachdem Helmut Schelsky ${ }^{2}$ in den Wirtschaftswunder «-Jahren die »nivellierte Mittelstandsgesellschaft « ausgerufen hatte und diese Formation dann in der »BolteZwiebel « ${ }^{3}$ ihr Piktogramm fand, wurde viele Jahre lang nicht mehr viel über die Mittelschichten gesprochen. Zwar war das relativ harmonische Selbstbild einer auf eine »Mittellage hin nivelliert[en] ${ }^{4}$ Gesellschaft nie unumstritten, aber sowohl normativ wie auch empirisch wurde die Vorstellung einer von mittleren Soziallagen dominierten Sozialstruktur zur wenig hinterfragten Selbstverständlichkeit. Auch deshalb sprach man in vielen Angelegenheiten die Mittelschichten nicht ausdrücklich an, weil sie - wie jeder wusste - stets irgendwie gemeint waren. Wenn zwei Drittel der Gesellschaftsmitglieder zu den Mittelschichten gerechnet wurden, musste man umgekehrt ausdrücklich vermerken, wenn man - wie im Diskurs der 1980er Jahre über die "Zweidrittelgesellschaft « - von den unteren sozialen Schichten und etwas später dann von der »Neuen Armut « oder gar den »Unterklassen « 5 als einem ganz anderen Teil der Gesellschaft redete oder umgekehrt, viel seltener, die Oberschicht der Reichen oder der Funktionseliten thematisierte. Die Mittelschichten galten als die breite Mehrheit derer, die nicht bloß dazugehören, sondern geradezu Repräsentanten dessen sind, was die deutsche Gesellschaft an Lebenschancen bietet.

Seit einigen Jahren sind nun die Mittelschichten - nicht nur in Deutschland, auch in anderen westlichen Gesellschaften wie den USA oder Frankreich - ein prominentes Thema gesellschaftlicher und sozialwissenschaftlicher Debatten. ${ }^{6}$ Diagnosen

1 Die Überlegungen dieses Beitrags sind in intensiven Diskussionen entstanden, an denen neben den Autoren weiterhin Sonja Drobnic, Karin Gottschall, Johannes Huinink und Michael Windzio beteiligt waren. Wir danken ihnen für die Mitwirkung an der Erarbeitung der hier formulierten Ideen. Von wem aus diesem Kreis welche Idee stammt, können wir im Einzelfall gar nicht mehr sagen. Für weitere Hinweise zu einer früheren Fassung des Beitrags danken wir ferner Fabian Gülzau, Nadine Schöneck-Voß, Ute Volkmann und Michael Walter sowie zwei anonymen Gutachtern.

2 Schelsky 1953.

3 Bolte 1966.

4 Schelsky 1953, S. 224.

5 Dahrendorf 1992.

6 Ehrenreich 1989; Chauvel 2006; Herbert-Quandt-Stiftung 2007; Bagnasco 2008; Vogel 2009; Vogel 2011; Collado 2010; Heinze 2011; Hacker, Pierson 2011; Mau 2012; Burkhardt et al. 2012; Gornick, Jäntti 2013; Koppetsch 2013; Fourquet et al. 2013.

Leviathan, 42. Jg., 2/2014, S. $219-248$ 
zum Schrumpfen der Mittelschicht, zur Abschottung nach unten oder zu neuen Abstiegsängsten sind häufig bediente, freilich kontrovers diskutierte mediale Aufreger, weil sie das heutige gesellschaftliche Selbstverständnis zentral berühren: Der - vermeintliche oder tatsächliche - Wandel zum Schlechten, den die Mittelschichten erleiden müssen, wird zur Chiffre für problematischen gesellschaftlichen Wandel insgesamt. Als gesellschaftliche Dynamiken, die diese Verunsicherungen der Lebensführung der Mittelschichten auslösen, werden vor allem die wirtschaftliche und kulturelle Globalisierung ${ }^{7}$, Entwicklungen der Prekarisierung und der Rückkehr der Unsicherheit ${ }^{8}$, der »Neoliberalismus « ${ }^{9}$, der $»$ neue Kapitalismus «10, der Finanzmarkt-Kapitalismus ${ }^{11}$ sowie eine allgemeine gesellschaftliche Beschleunigung ${ }^{12}$ ausgemacht.

Dies sind zunächst einmal die zumeist als Ursachen genannten Auslöser der Verunsicherungen. Unverkennbar hat die seit dem gesellschaftlichen Umbruch Mitte der 1970er Jahre einsetzende allgemeine gesellschaftliche Krisenrhetorik nun auch die Mittelschichten erreicht. ${ }^{13}$ Im Vordergrund steht hierbei vielfach ein simples Opfer-Narrativ: Die Mittelschichten werden von einem strukturellen Wandel erfasst und müssen Verluste an Sicherheit, Wohlstand, Lebenschancen und Einflussmöglichkeiten hinnehmen. Sie müssen auslöffeln, was ihnen andere, zum Beispiel die »Banker " oder die Sozialdemokraten oder anonyme gesellschaftliche Kräfte, eingebrockt haben. Dabei wird jedoch oft übersehen, dass die Mittelschichten selbst, wenngleich mitunter ungewollt, aktiv zu den gesellschaftlichen Dynamiken beigetragen haben, die sich nun auch für sie zu handfesten Problemen ausgewachsen haben könnten. Das Spektrum reicht von einer »aktiven Mittäterschaft « von Teilen der Mittelschichten an den eigenen Verunsicherungen (etwa als Pioniere neuer »flexibler « oder " partnerschaftlicher « Lebensstile, als spekulierende Kleinanleger, als ehrgeizige Reformer öffentlicher Verwaltungen, als auf Steuersenkungen pochende Wählerschaft oder als Bannerträger der Wettbewerbs- und Leistungsideologie) ${ }^{14}$ über eine passive Zustimmung der Mittelschichten zu »neoliberalen « Programmatiken (als "Marktvolk ", das dem Finanzkapital seine Ersparnisse für globale Operationen überlässt, und als »Wahlvolk «, das entsprechenden Parteiprogrammen seine Stimme gibt) ${ }^{15}$ bis hin zu Formen der » unterlassenen Hilfeleistung « (etwa als die Mittelschichten die "Montagsdemonstrationen « gegen den Sozialstaatsabbau der Hartz-IV-Reformen den »einfachen Leuten « überließen). Es wäre angesichts der Größe dieser Bevölkerungsgruppe und ihrer Stellung im gesamtgesellschaftlichen

7 Held 2000; Dürrschmidt 2002.

8 Castel, Dörre 2009.

9 Mudge 2008.

10 Boltanski, Chiapello 2003.

11 Windolf 2005.

12 Rosa 2006.

13 Zu diesem Umbruch siehe Doering-Manteuffel, Raphael 2008 sowie Jarausch 2008.

14 Cornelia Koppetsch (2013, S. 165-177) betont und illustriert derartige Phänomene.

15 Vgl. Streeck 2013. 
Gefüge ja auch absurd, anzunehmen, dass weitreichende gesellschaftliche Veränderungen ohne die aktive Beteiligung oder passive Duldung der Mittelschichten denkbar wären.

Wenn man davon ausgeht, dass die Mittelschichtangehörigen insgesamt in ihrem handelnden Zusammenwirken wichtige Treiber dessen sind, was sie je individuell und kollektiv mehr und mehr zu verunsichern scheint, muss man zuallererst fragen: Was ist die generelle Logik der Lebensführung der Mittelschichten? In welchen Mustern des Handelns je einzelner Mittelschichtangehöriger prägt sie sich aus? Erst auf dieser Grundlage lässt sich die weitere Frage danach stellen, welche Zusammenhänge zwischen heutigen gesellschaftlichen Veränderungsdynamiken auf der einen und Veränderungen der Lebensumstände und Lebensführung der Mittelschichten auf der anderen Seite bestehen.

Um keine falschen Erwartungen zu wecken, sei betont, dass wir hier kein umfassendes und empirisch gesättigtes Bild der heutigen Mittelschichten und ihrer Zukunftsaussichten zeichnen können, sondern zunächst einmal notwendige theoretische Vorarbeiten als Grundlage noch zu leistender, anders als bisher angelegter empirischer Untersuchungen zur Diskussion stellen.

\section{Die Lebensführung der Mittelschichten: Ein theoretisches Modell}

Die Frage, was die Mittelschichten ausmacht, steht im Kontext historisch weit zurückgehender gesellschaftspolitischer und sozialwissenschaftlicher Erörterungen, deren soziologischer locus classicus Max Webers ${ }^{16}$ Protestantismus-These ist, die der » methodischen Lebensführung « bestimmter religiöser Gruppen als Teil der bürgerlichen Mittelschichten eine entscheidende Rolle bei der Heraufkunft des modernen Kapitalismus als Kern des »okzidentalen Rationalismus « zuspricht. Dies gilt auch für die nach dem Zweiten Weltkrieg an Weber anschließende Modernisierungstheorie ${ }^{17}$, die freilich die Ambivalenzen der damit einhergehenden Gesellschafts- und Lebensform einseitig zum Positiven verharmloste. Während der »okzidentale Rationalismus « bei Weber im "stahlharten Gehäuse der Hörigkeit « zu enden droht, stehen die Mittelschichten gemäß der modernisierungstheoretischen Lesart für Demokratie und politische Stabilität, für marktwirtschaftliche Dynamik und Wohlstand, für wissenschaftliche Innovationskraft und Bildungsexpansion sowie nicht zuletzt für individuellen Aufstiegswillen - kurz: für die dynamische Modernität der Moderne.

Dieses optimistische modernisierungstheoretische Narrativ, das auch heute noch auf internationaler Ebene die Diskussionen um die "emerging middle classes" begleitet, hat sich im Westen insbesondere bis zur Mitte der 1970er Jahre ausgeprägt. In dieser Zeit haben die Mittelschichten in Deutschland den Durchbruch von einer bedeutsamen, schon früher gelegentlich als Zünglein an der Waage fungierenden Minderheit zur dominierenden sozialstrukturellen Großgruppe vollzogen.

16 Weber 1975 [1905].

17 Lerner 1958; Berger 1996.

Leviathan, 42. Jg., 2/2014 
Spätestens ab Mitte der 1980er Jahre konnte man faktisch deutlich mehr als die Hälfte der Bevölkerung in dieser Kategorie verorten, ${ }^{18}$ wenn man, wie weit verbreitet, beispielsweise einen mittleren Bildungsabschluss und ein mittleres Einkommen als Kriterien zugrunde legt.

Was in Webers Betrachtung noch klar im Vordergrund stand, die durch eine übergreifende Logik gestiftete Ganzheitlichkeit der Lebensführung in den verschiedenen Lebensbereichen und der Vielfalt konkreter Praktiken, ist in den sozialwissenschaftlichen Untersuchungen der Mittelschichten seit Längerem in den Hintergrund gerückt. Soziologische sowie wirtschafts- und politikwissenschaftliche Analysen der Mittelschichten haben mittlerweile eine Fülle an Befunden zur Ausstattung dieser Bevölkerungsgruppe mit verschiedenen Arten von Ressourcen und zu Veränderungen dieser Ressourcenbasis, die der gesellschaftliche Wandel verursacht hat, vorgelegt; ebenso weiß man inzwischen viel über Handlungsweisen von Mittelschichtangehörigen in den verschiedensten Lebensbereichen, vom Bildungsbis zum Gesundheits-, vom Wahl- bis zum Konsumverhalten. Anders gesagt: Die Lebenschancen und die bereichsspezifischen Handlungsmuster der Mittelschichten sind relativ gut bekannt. Doch zur bereichsübergreifenden Lebensführung, wie sie sich aus der Ressourcenausstattung im Kontext ermöglichender und limitierender institutioneller Strukturen ergibt, bietet die heutige Forschung, verglichen mit dem prägnanten Porträt des asketischen Protestanten bei Weber, nur noch eine blasse, analytisch nicht durchdrungene Schattenfigur. Auch die Lebensstilgruppierungen oder Milieus, die die kulturalistischen Spielarten der Ungleichheitssoziologie herausarbeiten, ${ }^{19}$ bleiben weitgehend in additiven Listen empirisch identifizierter Charakteristika zum Beispiel der » modernen Performer « im Vergleich zu den »KonsumMaterialisten " - um zwei der sogenannten "Sinus-Milieus « anzuführen - stecken; eine die verschiedenen Charakteristika zusammenführende Logik der Lebensführung ist meist kaum erkennbar. Selbst Pierre Bourdieus ${ }^{20}$ großangelegtes Porträt der Sozialstruktur Frankreichs in den 1960er Jahren, das wie keine andere Analyse den Nexus von Ressourcenausstattung - in Gestalt ökonomischen, kulturellen und sozialen Kapitals - und Habitus mit besonderer Betonung der Mittelschichten hervorhebt ${ }^{21}$ verliert sich im Detailreichtum der lebensbereichsspezifischen »feinen Unterschiede " und gelangt bezüglich des Lebensführungsmodus von Mittelschichtangehörigen zu zweifellos scharf beobachteten, aber theoretisch wie normativ einseitigen und nicht hinreichend erläuterten Aussagen, wenn er etwa dem »Kleinbürgertum ", wie er die Mittelschichten insgesamt tituliert, einen Habitus der »Prätention « zuspricht.

Das angesichts dieses hier nur ganz knapp angedeuteten Forschungsstands von uns im Folgenden vorgeschlagene Verständnis der Mittelschichten versucht, Webers

18 Nicht nur in Deutschland, auch in anderen westeuropäischen Ländern - siehe Allum 1995.

19 Schulze 1992; Rössel, Otte 2011.

20 Bourdieu 1979.

21 Für Deutschland ist eine solche Herangehensweise in einigen der milieutheoretischen Ansätze weitergeführt worden; siehe Vester et al. 2001. 
Augenmerk auf Lebensführung wieder an den Anfang der Betrachtung zu stellen, und bedient sich bei der Charakterisierung des Lebensführungsmodus der Mittelschichten wichtiger theoretischer Konzepte Bourdieus. Entscheidend für unser Verständnis der Mittelschichten ist ein Nexus von Ressourcenausstattung und Lebensführung: Eine bestimmte Ausstattung der Mittelschichtangehörigen mit ökonomischem und kulturellem Kapital passt zu einer Art der Lebensführung, die in einer permanenten Investition beider Kapitalsorten in Statuserhalt und -verbesserung besteht, was nun in einem theoretischen Modell auszuführen ist. Um Missverständnissen vorzubeugen, sei vorausgeschickt, dass dieses Modell ganz gezielt idealtypisch auf den kleinsten gemeinsamen Nenner der Lebensführung der Mittelschichten hin stilisiert ist; alle real vorkommenden und bedeutsamen Varianzen bleiben vorerst ausgeblendet.

\subsection{Investitionskalkül}

Gängigen Definitionen folgend, verfügen die Mittelschichten über eine mittlere Ausstattung mit Ressourcen, wobei das ökonomische und kulturelle Kapital im Vordergrund stehen. Das ökonomische Kapital stellt den disponiblen Geldbesitz in Gestalt von regelmäßigem Einkommen und bereitstehendem Geldvermögen dar, aber auch den Besitz von Immobilien, Wertgegenständen, Anwartschaften (etwa der Rentenversicherung) und schließlich Produktionsmitteln. Beim kulturellen Kapital geht es zum einen um Bildungs- und Ausbildungsabschlüsse, zum anderen um den durch soziale Herkunft, die Bildungslaufbahn sowie weitere Lebenserfahrungen angeeigneten Habitus. Sowohl Bildungszertifikate als auch der Habitus sind auf dem Arbeitsmarkt und für innerorganisatorische Karrieren verwertbar, nicht zuletzt auch für eine Praxis der Distinktion.

Entscheidend ist nun, dass die Kapitalausstattung einen bestimmten, die Mittelschichten auszeichnenden Modus der Lebensführung zwar keineswegs determiniert, jedoch nahelegt. ${ }^{22}$ Denn mittlere Kapitalausstattung bedeutet: Man hat einerseits so viel, dass man etwas zu verlieren hat; andererseits reichen das ökonomische und kulturelle Kapital dafür aus, dass man etwas investieren und daraus im weitesten Sinne Erträge erzielen kann. Das ist die sich aus dieser Kapitalausstattung ergebende Logik: Etwas gewinnen, aber auch etwas verlieren zu können, hält zum permanenten Investieren in den eigenen Status an. Zugespitzt: Zu den Mittelschichten gehört, wer genug hat, um mehr daraus machen zu können - und zu wenig, um nichts tun zu müssen. Noch anders gesagt: Er muss investieren - und er darf hoffen, damit Erfolge zu erzielen.

Wir bezeichnen den typischen Lebensführungsmodus der Mittelschichten daher als »investive Statusarbeit «, weil er charakterisiert ist durch das Bestreben, den

22 Dieser Modus muss nicht bewusst sein, sondern liegt in Gestalt des Habitus größtenteils als implizites Wissen vor, also als Wahrnehmungs-, Bewertungs-, Entscheidungs- und Handlungsdispositionen. Der Habitus ist somit zum einen Teil des kulturellen Kapitals, zum anderen das in der Lebensführung sich manifestierende Ergebnis des Zusammenwirkens von verfügbarem kulturellen und ökonomischen Kapital (Rehbein, Saalmann 2009 a; Rehbein, Saalmann 2009 b).

Leviathan, 42. Jg., 2/2014 
durch die jeweilige Kapitalausstattung bestimmten sozialen Status durch investive Praktiken zu erhalten und wenn möglich zu vermehren, und dies zumeist nicht nur individuell über den eigenen Lebensverlauf, sondern auch in der Generationenfolge. Dabei werden Ressourcen wie Geld und Bildung in dem Maße zu »Kapitalien «, wie sie investiv, also in die Zukunft gerichtet und mit Erwartung eines späteren Gewinns eingesetzt werden.

Das bedeutet zunächst: Alle Arten der Nicht-Investition beider Kapitalsorten dürfen in der Lebensführung von Mittelschichtangehörigen nicht überhandnehmen. Beim ökonomischen Kapital ist damit vor allem dessen übermäßige Verausgabung im unmittelbaren Konsum gemeint, um sich hier und jetzt ein "schönes Leben « zu ermöglichen. Denn dieses Geld kann dann nicht mehr investiv verwandt werden. Die Nicht-Investition kulturellen Kapitals besteht hingegen darin, davon zu zehren, ohne es zumindest zu erneuern. Denn kulturelles Kapital unterliegt immer wieder eintretenden Entwertungen, etwa durch den Wandel von Qualifikationsanforderungen der Arbeit oder durch neue kulturelle Trends, die den Distinktionswert bisheriger Praktiken mindern. Wer zum Beispiel mit der seit den 1980er Jahren pausenlos voranschreitenden Computerisierung sehr vieler Berufe nicht Schritt gehalten hat, war als Arbeitnehmer bald nicht mehr zu gebrauchen; und wer als Cineast um die Jahrtausendwende aufgehört hat, neue Filme anzuschauen, weil ihn seitdem andere Dinge des Lebens präokkupiert haben, kann nicht nur unter Kennern längst nicht mehr mitreden.

Mittelschichtangehörige müssen somit ihr ökonomisches und kulturelles Kapital investieren. Hier ist nun, Webers Stichwort der »rationalen Temperierung « ${ }^{23}$ des modernen kapitalistischen Erwerbstriebs aufgreifend, zwischen riskanten und vorsichtigen Investitionen zu unterscheiden. Riskante Investitionen bestehen darin, große Geldsummen oder sogar sein gesamtes Vermögen als Unternehmer, Investor oder auch Roulettespieler einzusetzen oder all sein kulturelles Kapital auf die Karte einer wissenschaftlichen oder künstlerischen Karriere - einschließlich Showbusiness - oder einer politischen Karriere setzen. Wenn dieses Hasardspiel glückt, kann man einen Sprung nach oben, vielleicht sogar in die Oberschichten machen. ${ }^{24}$ Aber viele scheitern bei diesem Versuch auch deshalb, weil sie in »winner takes all «-Konstellationen hineingeraten, in denen sich Gratifikationen nicht gemäß den tatsächlich vorliegenden graduellen Leistungsdifferenzen verteilen, sondern ein Gewinner alles bekommt. Die bei solchen Hochrisiko-Investitionen Gescheiterten können dann ebenfalls dort landen, wo sich diejenigen befinden, die nur von ihrem Kapital gezehrt haben.

Das der Kapitalausstattung der Mittelschichten adäquate, gewissermaßen »vernünftige « Investitionskalkül besteht demgegenüber überwiegend in vorsichtigen Investitionen: einem planvollen und fleißigen Immer-wieder-Nachschießen von Investitionen, um so auf längere Sicht den sozialen Status zu halten und möglichst

23 Weber 1963 [1919], S. 4.

24 Was Sighard Neckel (2008) als derzeitigen Wandel von der »Leistungs-« zur »Erfolgsgesellschaft « diagnostiziert, beschreibt die Haltung derer, die diese Investitionsoption wählen, sehr gut. 
sogar schrittweise zu steigern. Für diese Zwecke wird ökonomisches Kapital so eingesetzt, dass etwa das eigene Geschäft, das Aktiendepot oder der Immobilienbesitz ausgebaut wird; und das kulturelle Kapital wird vor allem mit Blick auf berufliche Verwertbarkeit und Karriere aktuell gehalten und ausgebaut, beispielsweise durch die Wahrnehmung von Weiterbildungs- und kulturellen Angeboten oder durch Anstrengungen des Lernens und Aneignens von Wissen und Kompetenzen.

\subsection{Kulturelle Weichenstellungen}

Im Kalkül vorsichtigen Investierens manifestiert sich zunächst die Interessenlage, die mit einer mittleren Kapitalausstattung verbunden ist. Welche konkreten Praktiken diesen Interessen entsprechen, hängt vor allem von institutionellen Gegebenheiten und der »stumme[n] Macht der Möglichkeiten « $25 \mathrm{ab}$, also zum Beispiel vom Arbeitsrecht und der Arbeitsmarktlage. Darauf ist noch zurückzukommen. An dieser Stelle ist erst einmal darauf einzugehen, dass die Ausformung der Statusinteressen dadurch ihre Richtung erhalten hat und beibehält, dass kulturelle Ideen als »Weichensteller « ${ }^{26}$ wirken. Das geschilderte Investitionskalkül hat sich historisch als für die Mittelschichten identitätsprägende »Rationalisierung der Lebensführung « ${ }^{27}$ mit bestimmten Mentalitäten, Deutungsmustern, Einstellungen und Maximen herausgebildet, die sich in den Praktiken der »alltäglichen Lebensführung « 28 darstellen. Hier sind die wohlbekannten Charakterisierungen der Mittelschichten zu nennen - Porträtelemente, die immer auch ein Stück weit idealisieren und derart fürs Selbstporträt benutzt werden. So wird die Mittelschicht als leistungs- und aufstiegsorientiert gesehen und sieht sich auch selbst so; sie ist bereit zum Bedürfnisaufschub und investiert in Bildung, Eigentum, Vorsorge; sie nimmt eine bewusste Berufswahl vor und engagiert sich, um Karrierechancen zu wahren; sie folgt institutionell vorgezeichneten, aber in diesem Rahmen Entscheidungsspielräume lassenden Lebenslaufmustern von Qualifikationserwerb, ökonomischer Unabhängigkeit von den Eltern, Heirat, Elternschaft und Bildungslaufbahnen der Kinder, die dann wieder den Staffelstab mit eigenen schulischen Anstrengungen übernehmen.

Die beiden in der "Kultur der Moderne ${ }^{29}$ verankerten tragenden Säulen dieser auf Statussicherung und -ausbau ausgerichteten Lebensführung sind zum einen ein Leistungsethos, zum anderen ein Planungsimperativ. Die Mittelschichten sind die Trägergruppe der Legitimation sozialer Ungleichheiten, die der in der Moderne aufgekommenen Gleichheitsidee allein noch trotzt: Leistung darf und muss sich lohnen! Nur wer sich gemäß dieser meritokratischen Vorstellung und entlang des vorherrschenden Verständnisses von Leistung mehr als andere anstrengt, hat seine besseren Lebenschancen redlich verdient. Hierbei dürfen die Möglichkeiten sozialen Aufstiegs nicht gedeckelt werden. Auch wer zum Beispiel als mittlerer Angestellter

25 Esser 2000, S. 269.

26 Weber 1963 [1919], S. 252.

27 Weber 1975 [1905], S. 165.

28 Voß 1991.

29 Münch 1986.

Leviathan, 42. Jg., 2/2014 
beginnt, kann es zum Topmanager schaffen. Aus derselben Logik heraus müssen die Mittelschichten dann auch nach unten hin offen für soziale Aufsteiger sein, zumal dann, wenn sich diese am die Mittelschichten prägenden Lebensführungsmodus ausrichten. Spätestens an diesem Punkt zeigt sich das Ideologische des Leistungsdenkens: Solange das Pochen auf Leistung dazu dient, Schranken des eigenen Aufstiegs zu delegitimieren, wird darauf insistiert; sobald hingegen unwillkommene Konkurrenz von unten auf den Plan tritt, werden mehr oder weniger subtile Argumente herangezogen, um faktische Bestrebungen einer sozialen Schließung nach unten zu legitimieren. ${ }^{30}$ So gehen Mittelschichteltern - um nur dieses augenfällige Beispiel zu nennen - manchmal im wahrsten Sinne des Wortes auf die Barrikaden, damit Schulformen wie das Gymnasium und der Zugang dazu auch weiterhin so ausgestaltet sind, dass vielleicht nicht so begabte und weniger fleißige Mittelschichtkinder möglichst keine Konkurrenz durch begabtere und fleißigere Kinder aus den Unterschichten fürchten müssen.

Ähnlich ambivalent - diesmal mit Blick auf die Mittelschichten selbst - stellt sich der Planungsimperativ dar. Auf der einen Seite sind die Mittelschichten diejenigen, die - das ist bereits im Leistungsethos impliziert - Erfolgschancen ihrer Statusinvestitionen nicht nur glücklichen Zufällen überlassen dürfen, sondern sie durch ein möglichst planvolles Vorgehen verbessern wollen. Auf der anderen Seite werden Lebensverläufe trotz aller Lebensplanung faktisch doch oft durch so viele Kontingenzen beeinträchtigt, immer wieder gestört oder regelrecht aus der Bahn geworfen, dass verfehlte Planziele regelmäßig durch Ex-post-Rationalisierungen vergessen gemacht werden müssen, um weiter an der Selbstzumutung, das eigene Leben planbar zu gestalten, festhalten zu können. ${ }^{31}$

Der charakteristische Duktus dieser Lebensführung lässt sich zusammenfassend auch im zugespitzten Kontrast zu den anderen beiden gesellschaftlichen Großgruppen aufzeigen. Die Kapitalausstattung der Unterschichten, also etwa derer, die in ungelernten oder angelernten Berufen tätig sind, ist oft zu gering, um Nennenswertes zum Investieren übrig zu haben. Zudem findet ihr entsprechendes Bemühen gesellschaftlich oft wenig Anerkennung, weil es sich anders darstellt oder auch nur als anders wahrgenommen wird. Ihre Lebensführung ist als eine »von der Hand in den Mund « charakterisiert worden, die zunächst einmal eher durch einen Mangel an Ressourcen als durch die Verfügung über sie bestimmt ist. Die unteren Schichten haben daher nur begrenzte Möglichkeiten des sozialen Aufstiegs und der Mehrung ihrer geringen Ressourcen, soweit sie überhaupt die Intention haben. Eine substanzielle Ressourcensteigerung und die Investition von Geld oder Bildung als Kapital ist, von glücklichen Umständen abgesehen, nur durch große Entbehrung möglich und damit weder als Selbstanspruch noch empirisch wahrscheinlich. Im Hinblick auf die von uns pointierte Passung zwischen Ressourcenausstattung und Lebensführungsmodus würden wir erwarten, dass der investive Modus zwar auch in den unteren Schichten vorkommen kann, aber nur nachhaltig und anhaltend verfolgt

30 Münkler 2010, S. 70.

31 Dies ist der vielleicht wichtigste Aspekt dessen, was Bourdieu (1986) als »biographische Illusion « bezeichnet. 
wird, wenn er in absehbarer Zeit entsprechende Statuserträge generiert, zum Beispiel ein Aufschließen zur Mittelschicht ermöglicht.

Demgegenüber bedeutet der - überspitzt gesagt - »sorgenfreie Reichtum «32 der Oberschichten nicht nur, dass sie ihr Leben gelassener als die stets besorgten Mittelschichten führen können, sondern auch, dass sie ihr ökonomisches Kapital in ungleich größerem Maßstab und mit höherem Risiko als die Mittelschichten investieren können, was Chancen bietet, die Kapitalausstattung noch weiter steigern zu können, ohne dabei auf standesgemäßen Konsum verzichten zu müssen. Wenn die Kapitalrendite zudem langfristig über dem Zuwachs bei den Einkommen liegt, ${ }^{33}$ ist es für Oberschichtangehörige sogar wichtiger, ihr Vermögen geschickt zu investieren und zu mehren, als ihr Humankapital andauernd zu erweitern - wobei sich beides nicht ausschließen muss. Auf den Punkt gebracht heißt das: Die Mittelschichten können und müssen permanente Statusinvestitionen tätigen, Unterschichten können es kaum, Oberschichten müssen es nicht, können und tun es aber zumeist. ${ }^{34}$

\subsection{Status-Bewegungen: Mobilitätsbemühungen und Kämpfe um » Wechselkurse"}

Bis hierher haben wir den idealtypischen einzelnen Mittelschichtangehörigen und dessen Prinzipien und Praktiken der Lebensführung in den Blick genommen. Wie stellt sich nun eine solche Lebensführung im gesellschaftlichen Kontext dar, in dem sich viele andere Mittelschichtangehörige mit gleicher Lebensführung ebenso wie die Angehörigen der Ober- und Unterschichten mit ihren je spezifischen Lebensführungsmustern bewegen?

Zum einen muss sich jeder einzelne Mittelschichtangehörige im Rahmen der sich ihm bietenden Möglichkeiten für den Erhalt oder die Steigerung seines Kapitals bewegen. Hierbei passt sich das Individuum den Verwertungsbedingungen so gut wie möglich an. Parallel zu dieser je individuellen Anpassung an gegebene Strukturen und das Ergreifen sich bietender Chancen bemühen sich weiterhin auch Gruppen von Individuen mit ähnlichen Interessen darum, gezielt bestimmte Strukturen zu den eigenen Gunsten umzugestalten. Das findet sich bei den Mittelschichten in besonderer Weise, die in hohem Maße diejenigen Berufs- und Interessengruppen stellen, die sich in die Gestaltung der gesellschaftlichen Rahmenbedingungen der individuellen Kapitalverwertung einschalten. Die Mittelschichten kontrollieren damit nicht nur aufgrund ihrer zahlenmäßigen Stärke als Wähler- und Interessengruppen, sondern auch als Experten, deren Einschätzungen zu Rate gezogen werden, in erheblichem Maße die Verwertungsbedingungen des kulturellen Kapitals und bis zu einer bestimmten Größenordnung auch die des ökonomischen Kapitals - wobei das große Kapital fest in der Hand der Oberschichten liegt. Ferner gestalten Mittelschichtangehörige in unterschiedlichen Rollen mit: in Leistungs- oder Publikumsrollen oder als politisch Engagierte, die sich in Interessenverbänden oder Bürgerinitiativen

32 Groh-Samberg 2009.

33 Picketty 2014.

34 Müller 1992, S. 375.

Leviathan, 42. Jg., 2/2014 
zusammenschließen. Dabei stehen sie auch untereinander in Konkurrenz- oder Konfliktbeziehungen - wenn etwa Patienten gegen Ärzte oder Ärzte gegen Pflegekräfte agieren.

Zunächst zur je individuellen Anpassung in Gestalt von Mobilitätsbemühungen: Deren Erträge hängen stark von drei gesellschaftlichen Kontextbedingungen ab. Die erste betrifft die Nachfrage nach den eigenen Qualifikationen, also bei Lohnabhängigen die Arbeitsmarktlage und bei Selbstständigen die Lage auf dem jeweiligen Güter- oder Dienstleistungsmarkt. Die Höhe und Struktur dieser Nachfrage bestimmt, in welchem Maße eine individuelle Statusverbesserung relativ zu Vergleichsgruppen möglich ist. Die mittelschichtspezifische Ambition besteht darin, auch bei einer ungünstigen Marktlage mindestens den Werterhalt des eigenen ökonomischen und kulturellen Kapitals und damit einen Statuserhalt zu erreichen, also eine »stationäre Reproduktion «. Bei günstigerer Marktlage nimmt man sich hingegen eine moderate Kapital- und Statussteigerung vor, also sozialen Aufstieg.

Parallel dazu wirkt als zweite Kontextbedingung die allgemeine Wirtschaftsentwicklung im Sinne eines kollektiven »Fahrstuhl-Effekts « 35 . Dieser kann sich - und das ist nicht erst seit den 1950er Jahren die Normalitätserwartung nicht nur der Mittelschichten - nach oben bewegen und mehr oder weniger alle gesellschaftlichen Gruppen mitnehmen. Immer wieder vorkommende Bewegungen nach unten werden - so die fortschrittsoptimistische Lesart der Moderne - als vorübergehend zu erduldende angesehen. Solange eine allgemeine Aufwärtsbewegung überwiegt, heißt dies aus der Perspektive der Mittelschichten, dass eine absolute Steigerung des eigenen Lebensstandards allein schon deshalb notwendig wird, um relativ zu anderen nicht zurückzufallen. ${ }^{36}$

Die individuelle Mobilität und der »Fahrstuhl-Effekt « wirken wie eine Vektoraddition zusammen. Beide Bewegungen können in die gleiche Richtung gehen, sich also aufaddieren, oder gegenläufig wirken und sich dann mehr oder weniger neutralisieren. Zu manchen Zeiten geht eine kollektive Verbesserung der Lebenschancen mit den Möglichkeiten individuellen Aufstiegs einher, wie es für viele Mittelschichtangehörige in Deutschland zwischen den 1950er und den 1980er Jahren der Fall war. Das andere Extrem sind gesellschaftliche Phasen einer allgemeinen wirtschaftlichen Depression, die für viele schwierigere Vermarktungsbedingungen ihrer beruflichen Qualifikationen mit sich bringt und auch zum Abbau sozialstaatlicher Leistungen führen kann; dann erleben viele einen sozialen Abstieg, und das auch noch in einem »Fahrstuhl « nach unten. Ebenso sind gegenläufige Bewegungen möglich, also individueller Aufstieg in Zeiten der Stagnation oder Abstieg trotz günstiger Rahmenbedingungen.

Als dritter, die Effekte der beiden anderen Faktoren moderierender - teilweise dämpfender, teilweise verstärkender - Kontextfaktor ist das Angebot an sozialer Sicherheit und darüber hinausgehenden wohlfahrtsstaatlichen Leistungen etwa im

35 Beck 1986, S. 122, Hervorhebungen weggelassen.

36 Es sei denn, der dauerhafte »Fahrstuhl-Effekt « nach oben genügt einem. Dann steigt man aus der Statusarbeit aus und vermag dennoch hinsichtlich der eigenen Kapitalausstattung Mittelschichtangehöriger zu bleiben. 
Bildungsbereich oder bei der Förderung von Wohneigentum zu nennen. Die Gewährung von Sicherheitsleistungen durch sozialpolitische Arrangements sorgt dafür, dass vorhandene Risiken abgepuffert und kompensiert werden, man also nicht befürchten muss, im Extremfall ins Bodenlose zu fallen. Dies ist einerseits eine institutionelle Voraussetzung für Individualisierung und eine größere Risikobereitschaft, was beides die Statusinvestitionen der Mittelschichten mitträgt. Andererseits kann auch nicht ausgeschlossen werden, dass gesteigerte Sicherheiten ab einem gewissen Punkt die Investitionsbereitschaft schwächen, man sich also - wie man es Teilen der Unterschichten immer wieder vorhält - in der »sozialen Hängematte " ausruht.

Die jeweilige Ausgestaltung dieser drei Kontextbedingungen, die die Spielräume individueller Chancennutzung von Mittelschichtangehörigen bestimmen, ist ihrerseits das Resultat vielfältiger Aktivitäten kollektiver und korporativer Akteure in der gesamtgesellschaftlichen Konstellation. Zu diesen Aktivitäten gehört die individuelle Mitwirkung an kollektiven Kämpfen von Mittelschichtgruppierungen um eine für sie vorteilhafte Gestaltung der Kontextbedingungen der Statussicherung. Weil sehr oft Vorteile der einen - zumindest als solche wahrgenommene - Nachteile anderer bedeuten, geht es dabei um Auseinandersetzungen, in denen Mittelschichtangehörige zum einen gegen Interessen der Ober- oder Unterschichten, zum anderen und nicht zuletzt aber auch gegen Interessen anderer Mittelschichtfraktionen auftreten.

Die für den Lebensführungsmodus der Mittelschichten konstitutive mittlere Ausstattung mit ökonomischem und kulturellem Kapital weist ja in sich eine beträchtliche Bandbreite auf; und je nach Kapitalausstattung stellt sich die Umsetzung des geschilderten generellen Investitionskalküls der Mittelschichten unterschiedlich dar. So kann es beim erworbenen kulturellen Kapital um den Marktwert spezifischer Bildungsgänge und -abschlüsse gehen, wenn etwa die Marktchancen technischer Berufsabschlüsse gegenüber kaufmännischen Abschlüssen steigen oder wenn Berufsgruppen wie das medizinische Pflegepersonal sich akademisieren, um so ein kollektives Statusprojekt zu verfolgen. Viele bildungs-, aber auch familienpolitische Auseinandersetzungen drehen sich ebenso wie Statuskämpfe zwischen Berufen darum, dass bestimmte Arten kulturellen Kapitals auf- oder abgewertet werden. ${ }^{37}$ Beim ökonomischen Kapital geht es, wie bereits erwähnt, um ganz verschiedene Arten von Geldbesitz und -quellen. Steuerpolitische Auseinandersetzungen ebenso wie die Debatten um die "richtige " Wirtschafts- und Sozialpolitik sind letztlich Manifestationen von Kämpfen darum, welche Arten von ökonomischem Kapital wem wie viel wert sind. Verallgemeinert: Da alle Fraktionen der Mittelschichten ihre Lebensführung mit ihrem jeweiligen Portfolio der verschiedenen Arten von ökonomischem und kulturellem Kapital betreiben, stellen die zum einen internen, zum anderen mit den Ober- und Unterschichten geführten Kämpfe um die »Wechselkurse « dieser fraktionsspezifischen »Währungen " gleichsam das Gruppenbild der Mittelschichten dar, das sich aus dem zuvor gezeichneten Einzelporträt des typischen Mittelschichtangehörigen ergibt.

37 Andrew Abbotts (1988) professionssoziologische Perspektive betont solche Statuskämpfe als Movens gesellschaftlicher Dynamiken. 


\section{Mittelschichten und gesellschaftliche Dynamik: Eine zeitdiagnostische Forschungsagenda}

Wie einleitend angesprochen, kursieren derzeit Befürchtungen, dass die «Mitte « vieler westlicher Gesellschaften vor zunehmenden Existenzproblemen stehe oder gar in eine tiefe Krise geraten sei. Zeitungs-Schlagzeilen verkünden: »Deutschlands Mittelschicht: vom Aussterben bedroht ${ }^{38}$ - ohne Fragezeichen! Zurückgeführt wird dieser vermeintliche Niedergang auf vielfältige und in ihrem Zusammenwirken schwer berechenbare gesellschaftliche Dynamiken, vom technologischen und wirtschaftlichen Wandel bis hin zum Wertewandel. Wenn sich aber gesellschaftliche Dynamiken als Veränderungen der Lebensführungsbedingungen der Mittelschichten auswirken und zugleich umgekehrt gilt, dass diese inzwischen selbst, allein schon aufgrund ihrer Größe, eine wichtige - und sich teils dessen bewusste - Trägergruppe solcher Dynamiken ist, bietet es sich an, dieses Wechselspiel in drei Analyseschritten zu modellieren: ${ }^{39}$

- Ausgangspunkt ist die Betrachtung der Situation, in der sich Akteure befinden. Hier stehen - so unsere theoretische Synthetisierung von teilweise bereits empirisch gut aufgearbeiteten Phänomenen - die durch strukturellen Wandel in der Gesamtgesellschaft hervorgerufenen Irritationen der Lebensführung der Mittelschichten im Vordergrund.

- Hieran schließt die Untersuchung der Handlungswahl an: Mit welchen Praktiken reagieren Mittelschichtangehörige auf die Irritationen? Hier stellen wir eine Typologie von Praktiken der Irritationsbewältigung zur Diskussion, die eine weithin erst noch zu leistende empirische Forschung anleiten könnte.

- Wiederum daran anknüpfend, werden die Aggregationseffekte in den Blick genommen. Hier geht es um die Auswirkungen des massenhaften Handelns von Mittelschichtenangehörigen und anderen gesellschaftlichen Akteuren auf gesellschaftsstrukturelle Dynamiken. Dazu weiß man bislang empirisch und theoretisch am allerwenigsten. Wir schlagen vor, solche mittelschichtgetriebenen Strukturdynamiken als Zusammenspiel dreier Arten von Aggregationen zu modellieren.

Je weiter unsere Betrachtung voranschreitet, desto weniger gesichertes Wissen ist vorhanden, und wir müssen uns immer mehr darauf beschränken, Blickrichtungen mit Hilfe von Begriffsvorschlägen, Typologien und spekulativen Leithypothesen zu justieren. Deshalb sprechen wir von einem Problemaufriss, der seine Hauptfunktionen erfüllt, wenn er erstens plausibel zu machen vermag, dass die Mittelschichten ein wichtiger Gegenstand heutiger sozialwissenschaftlicher Analyse sind, und zweitens zentrale Elemente eines heuristischen Bezugsrahmens zur Diskussion stellt, der

38 Der Tagesspiegel vom 16. Dezember 2012.

39 Auch als »Coleman'sche Badewanne « bekannt - siehe dazu nur Esser (1993, S. 91-102). Dabei übernehmen wir nicht die mit diesem Modell oft verbundene Handlungstheorie der Rational Choice. Siehe auch Deutschmann (2005), der seine Analyse der Finanzmarktaktivitäten der Mittelschichten ebenfalls unter Nutzung dieses sozialtheoretischen Bezugsrahmens konzeptualisiert. 
sich dann in der empirischen Umsetzung und theoretischen Fortführung zu bewähren hat.

\subsection{Multiple Irritationen: Investitionsstörungen der Mittelschichten}

Vor dem Hintergrund des skizzierten theoretischen Verständnisses lassen sich die einleitend erwähnten Verunsicherungen der Mittelschichten systematischer fassen - nämlich als Irritationen der Lebensführung, insbesondere des Investitionskalküls. Wir sprechen hier bewusst neutral von Irritationen und nicht pauschal von Verunsicherungen. Irritationen sind vom Individuum wahrgenommene Störungen dessen, was es als normalen Ablauf typischer Praktiken seiner Lebensführung kennt und oftmals hochgradig habitualisiert hat; es registriert, dass - umgangssprachlich formuliert - »die Dinge nicht mehr so laufen wie bisher « ${ }^{40}$ Das kann zum einen heißen, dass sich die Verhältnisse tiefgreifend wandeln, zum Beispiel die Qualifikationsanforderungen von Berufsfeldern oder die Erfordernisse der Alterssicherung. Die Verhältnisse können aber auch strukturell gleich bleiben, allerdings anstrengender im Umgang werden, weil etwa die Konkurrenz auf Heiratsmärkten oder die Zeitknappheit zunimmt. Eine Irritation kann als problematisch eingestuft werden und wirkt dann in der Tat verunsichernd - beispielsweise eine Kündigung oder das an einem Seitensprung manifest werdende Sich-auseinander-Gelebt-Haben eines Paares. Eine Irritation kann aber auch einfach nur in dem Sinne stören, dass sie ein »Weiter so! « des Handelns als nicht opportun erscheinen lässt, aber die Person davon ausgeht, dass es genauso gute Alternativen gibt, die nur gefunden werden müssen - oder dass die Störung sogar eine gute Gelegenheit ist, eine bessere Alternative zu finden.

Das Investitionskalkül der Mittelschichten ist vielfältig anfällig für Irritationen. Was seit den 1980er und verstärkt seit den 1990er Jahren an Unbehagen von Mittelschichtangehörigen mit der eigenen Lebenssituation geäußert wird, bezieht sich in der Tat durchgängig auf Irritationen, die neuralgische Punkte des Lebensführungsmechanismus betreffen. Acht, auch öffentlich viel diskutierte Störfelder sollen hier angesprochen werden. Es geht zunächst um sechs Lebensbereiche, in denen die Statusinvestitionen der Mittelschichten getätigt und inzwischen auf vielerlei Weisen irritiert werden:

- Arbeit: ${ }^{41}$ Die berufliche Arbeit und der Arbeitsmarkt bilden für die meisten männlichen ebenso wie weiblichen Mittelschichtangehörigen den zentralen gesellschaftlichen Ort ihrer Statusarbeit. Auf dem Arbeitsmarkt und bei den beruflichen Karrieren hat es in den vergangenen zwei Jahrzehnten strukturelle Veränderungen gegeben, die für viele Arbeitnehmer - nicht nur aus den Mittelschichten - Unsicherheiten gezeitigt haben. Treiber sind eine ganze Reihe von Entwicklungen wie die Globalisierung, der technologische Fortschritt, organi-

40 Irritationen gehen nicht nur von Veränderungen aus. Es kann ebenso irritieren, dass Lebensumstände geradezu erstarren, obwohl man sie sich immer dringlicher ganz anders wünscht - siehe etwa das »lock in « negativ bewerteter pfadabhängiger Dynamiken (Arthur 1989).

41 Heinze 2011; Mau 2012; Koppetsch 2013.

Leviathan, 42. Jg., 2/2014 
sationale Veränderungen, Flexibilisierung der Beschäftigungsverhältnisse, die Ausbreitung atypischer Beschäftigung und eine daraus hervorgehende Segmentierung der Arbeitsmärkte in ein Marktsegment für relativ gesicherte Insider und ein anderes Segment für eine wachsende Gruppe von prekären Outsidern. Im Zuge dieser Prozesse werden berufliche Positionen und Arbeitsmarktchancen neu bewertet, was ganz generell Irritationen auslösen kann, weil man sich eines einmal erreichten Status nicht mehr sicher sein kann. Zugleich kommt es für einige Mittelschichtfraktionen zu Verschlechterungen des Marktwerts ihrer Qualifikationen, mit der Folge von Unsicherheiten - vom Verlust als sicher unterstellter Karrierechancen bis hin zu Prekarisierung und Arbeitsplatzverlusten - und Einkommenseinbußen. Hinzu kommen ein gesteigerter Leistungsdruck sowie Mobilitäts- und Flexibilitätszumutungen, was alles auch Signale dafür sind, dass man ständig auf der Hut sein muss. Als Folge erfahren viele Mittelschichtangehörige neue Anforderungen an die aktive Selbststeuerung, die Selbstökonomisierung der Arbeitskraft, eine Entgrenzung von Arbeit und Privatleben sowie Zustände der Erschöpfung. ${ }^{42}$

- Partnerschaft: ${ }^{43}$ Die Partnerschaft - ob als Ehe oder als eheähnliches Zusammenleben - gilt zum einen als ein zentraler gesellschaftlicher Ort der temporären Suspension der Statusinvestitionen, ${ }^{44}$ zum anderen bildet sie in Gestalt von verbundenem Leben (»linked lives ${ }^{45}$ ) auch den eigentlichen korporativen Akteur und damit die Entscheidungsinstanz der Statusinvestitionen. Veränderungen der Geschlechterverhältnisse, insbesondere eine verstärkte Berufstätigkeit von Frauen, Vereinbarkeitsprobleme von beruflichen und familialen Anforderungen und erhöhte Ansprüche an die Qualität von Intimbeziehungen, sorgen in größeren Teilen der Mittelschichten für wechselseitige Verunsicherungen, die schon bei der Partnersuche beginnen und sich in vielen gemeinsamen Entscheidungen über Arbeitsteilung im Haushalt, Wohnarrangements, »dual career «-Planung oder Elternschaft fortsetzen. Eine deutliche Zunahme nicht-traditionaler Formen des Zusammenlebens bringt neue Erfordernisse der Aushandlung in Partnerschaften und Familien hinein und erzeugt so Instabilitäten, denn beide Partner können Unterschiedliches wollen. Dass beide Seiten, vor allem aber auch die Frauen, größere »exit «-Optionen haben, um mit Albert Hirschman zu sprechen, und auch nutzen, produziert mehr »voice ", also Konflikte, und weniger »loyalty « im Sinne der Akzeptanz der männlichen Lebensführung. ${ }^{46}$ All das bedeutet nicht nur erhöhte Koordinationserfordernisse und dementsprechend eine größere Gefahr drohender Entscheidungskonflikte für die Partnerschaft, sondern

42 Voß, Pongratz 1998; Neckel, Wagner 2013.

43 Solga, Wimbauer 2005; Illouz 2007; Buhr, Feldhaus 2012; Mau 2012; Wimbauer 2012; Koppetsch 2013.

44 Spätestens das Bürgertum des 19. Jahrhunderts postulierte dieses Verhältnis von Familienintimität und Berufswelt, mit den entsprechenden Zuständigkeitsaufteilungen an beide Geschlechter.

45 Rossi, Rossi 1990; Elder 1998.

46 Um Hirschmans (1970) bekannte Kategorien anzuwenden. 
läuft weiterhin darauf hinaus, dass diese auch als Ort, wo man temporär von den Anstrengungen der Statusarbeit "abschalten « kann, an Wirkungskraft einbüßt.

- Woblfahrtsstaat: ${ }^{47}$ Die Arbeitseinkommen als Resultate beruflicher Statusarbeit sind insbesondere seit den 1950er Jahren in Deutschland in erheblichem Maße durch wohlfahrtsstaatliche Leistungen abgesichert und auch aufgestockt worden. Über die vergangenen zwei Dekaden haben sich allerdings die Regelungen der wohlfahrtsstaatlichen Leistungen zuungunsten der Leistungsbezieher verändert, so etwa durch den Um- und Rückbau statussichernder Transferprogramme und die Verlagerung staatlicher Intervention weg von Kompensationsleistungen hin zu » investiven « Formen der Sozialpolitik, die dem Einzelnen ein Mehr an »Eigenvorsorge ", Beweglichkeit und Aktivierbarkeit abverlangen; ${ }^{48}$ und nicht nur sozialpolitische Leistungen, sondern auch staatlich getragene oder subventionierte Bildungs-, Kultur- oder Sportangebote, die die Statusarbeit vieler Mittelschichtangehöriger bislang unterstützt haben, werden zunehmenden Sparzwängen unterworfen, die sich im »Konsolidierungsstaat ${ }^{49}$ noch weiter verschärfen dürften.

- Vermögensbildung: ${ }^{50}$ Für die Mittelschichten spielt die Vermögensbildung als Form der Statusarbeit mit Blick auf ökonomisches Kapital eine immer schon wichtige Rolle. Zwar sind Vermögenseinkünfte anders als in den Oberschichten bislang im Vergleich zu Einkommen aus Arbeit noch nicht sehr gewichtig; ${ }^{51}$ aber zur längerfristigen Sicherung des Lebensstandards, insbesondere im Alter und angesichts des gerade erwähnten Abbaus des Wohlfahrtsstaats, und mit Blick auf den Aufbau generationenübergreifender Anlagen wird Vermögensbildung zu einer Voraussetzung für den Statuserhalt. Wesentliche Irritationen der letzten Jahre sind hier die unsicheren Erträge von Vorsorgestrategien auf einem turbulenten Finanzmarkt und ungünstige wirtschaftliche Entwicklungen gewesen.

- Elternschaft: ${ }^{52}$ Elternschaft ist schon immer eine langfristige biografische Festlegung gewesen, die zum einen tendenziell Restriktionen - nicht nur zeitlicher Art - der Statusarbeit nach sich zieht, zum anderen aber auch selbst Teil der Arbeit am Status darstellt. Soweit das traditionelle Modell der geschlechtlichen Arbeitsteilung und die Rollenzuweisung von männlichem Ernährer und weiblicher Mutter- und Hausfrauenrolle aufgebrochen werden, geht Elternschaft mit Aushandlungsprozessen im Dreieck von elterlicher Verantwortung, beruflichen Ambitionen und partnerschaftlichem Zusammensein einher. In relativ enger biografischer Taktung müssen ganz wesentliche Entscheidungen der Vereinbarkeit von Beruf und Familie getroffen werden, und dies mit sehr langfristigen und

47 Lessenich 2008; Vogel 2009; Heinze 2011.

48 Hacker 2006.

49 Streeck 2013.

50 Schimank 2011 a; Schimank, Stopper 2012.

51 Burkhardt et al. 2012.

52 Bude 2011. 
deshalb unüberschaubaren Folgen. Zugleich bedeutet Elternschaft auch den Schritt in die inter-generationale Statusarbeit: Kinder starten ihre Statusinvestitionen an dem Punkt, wo ihre Eltern sie durch Entscheidungen über deren Bildungslaufbahn platzieren. Mittelschichteltern sehen sich diesbezüglich zunehmend in der moralischen Pflicht, die Bildungslaufbahn ihrer Kinder von vorschulischen Förderungen bis zum akademischen Abschluss durch eigene Interventionen abzusichern, wovon der wachsende private Bildungsmarkt Zeugnis ablegt. ${ }^{53}$ Dies geschieht aber in harter Konkurrenz mit den anderen Mittelschichteltern, was den Erfolg der eigenen Bemühungen wiederum unsicherer werden lässt.

- Politisches Engagement: ${ }^{54}$ Wie bereits erwähnt, beinhaltet die Statusarbeit der Mittelschichten auch kollektive Bemühungen der Einflussnahme auf die politische Gesellschaftsgestaltung. Dass sich aber Regierungen, Parlamente und Parteien in den Augen eines Teils der Mittelschichten immer weniger um die Belange der Wähler zu kümmern scheinen und dort, wo sie es versuchen, an der Komplexität der Gestaltungsaufgaben zu scheitern scheinen, wird mit ohnmächtiger Beunruhigung registriert oder von »Wutbürgern « auch mit unkonventionellen Formen politischer Partizipation beantwortet. Der Anspruch auf Mitwirken ist nach wie vor vorhanden, aber oft scheint nicht einmal klar, an wen politische Forderungen noch sinnvoll gerichtet werden können. Im Zuge der Finanz- und Eurokrise hat sich diese Wahrnehmung noch einmal verschärft, da unter Bedingungen der Staatsverschuldung Finanzmarktakteure immer mehr in der Lage sind, Länder in Austeritätspolitiken hineinzuzwingen, und damit die Möglichkeiten politischer Gesellschaftsgestaltung - unter anderem im Sinne der Mittelschichten -immer mehr einschränken. ${ }^{55}$ Dies ist Teil einer Meta-Irritation darüber, dass man der aufgelisteten multiplen Irritationen immer weniger Herr zu werden vermag.

Alle genannten Lebensbereiche durchziehen zwei weitere Störfelder:

- Kulturelle Hegemonie: ${ }^{56}$ Als höchst förderliche ideelle Grundierung der Statusarbeit der Mittelschichten hat sich seit dem 18. Jahrhundert deren Deutungshoheit in Fragen eines "guten Lebens « und einer dieses ermöglichenden "guten Gesellschaft « erwiesen. Größere Teile der Mittelschichten sehen heute allerdings einen Bedeutungsverlust der von ihnen vertretenen bürgerlichen Werte, die sich um Leistungsethos und Planungsimperativ ranken. Sowohl Teile der Oberschichten, wenn sie sich als Topmanager zum Beispiel exorbitante Gehälter zuschanzen, als auch Teile der Unterschichten, insofern sie pauschal als faul und verwahrlost etikettiert werden, und last but not least auch andere Mittelschichtangehörige, wenn sie zu sehr dem Hedonismus frönen, werden für den »Werteverfall « verantwortlich gemacht; hinzu kommen Irritationen durch Prozesse

53 Knötig 2010; Newman 2012; Jungbauer-Gans et al. 2012.

54 Heinze 2011, S. 146-204; Marg et al. 2013.

55 Streeck 2013; Mau 2013.

56 Hradil, Schmidt 2007, S. 178-181, S. 211-222; Nolte, Hilpert 2007, S. 75-86. 
der Zuwanderung und Transnationalisierung. Die Debatten um die »neue Bürgerlichkeit " und die darin betonten Werte wie Verantwortung, Familie und Engagement sind angesichts dessen Versuche, nicht nur die Exklusivität, sondern auch die Definitionsmacht der Mittelschichten über kulturelle Werte aufrechtzuerhalten. ${ }^{57}$

- Körperlichkeit: Statusarbeit hat nicht nur ein kulturelles Dach, sondern auch ein körperliches Fundament. Auch diesbezüglich gibt es eine gesteigerte Verunsicherung von Mittelschichtangehörigen. Auf der einen Seite werden körperliche Fitness und psychosomatische Robustheit als unerlässlicher Teil dessen proklamiert, was ihnen nicht nur im Beruf abverlangt wird. Mittelschichtangehörige sehen sich einerseits mit Forderungen nach einem gesunden Lebensstil konfrontiert, der Sport-Treiben ebenso wie achtsame Ernährung verlangt und sich vor allem auch an einem Körperbild abzeichnen soll, das Vitalität und Belastbarkeit signalisiert. ${ }^{58}$ Doch auf der anderen Seite sind die faktischen Lebensumstände nicht nur im Beruf - dafür eher abträglich. Auch das artikulieren die Debatten über einen Ausgleich zwischen Privatleben und Berufsleben (»work-life balance «) und der Verweis auf gesellschaftliche Erschöpfungszustände. ${ }^{59}$ Jedes dieser - hier nur stichwortartig umschreibbaren - Störfelder müsste empirisch genauer ausgeleuchtet werden. Dabei wäre auch den vielfältigen Wechselwirkungen zwischen Irritationen in unterschiedlichen Störfeldern nachzugehen. So vernichtet oder verschlechtert zum Beispiel der Abbau des Wohlfahrtsstaats nicht zuletzt Mittelschicht-Arbeitsplätze, und die Entgrenzung von Arbeit, also das Hineinreichen beruflicher Anforderungen in das Privatleben, belastet Partnerschaften zusätzlich. Betont sei auch nochmals, dass unsere Sondierung der Störfelder Irritationen nur negativ thematisiert hat; denn so wirken sie in den aktuellen Mittelschichtdebatten. Dabei wäre zum einen empirisch zu klären, welche Teile der Mittelschicht wie stark von den oft nur mit anekdotischer Evidenz behaupteten Irritationen betroffen sind. Zum anderen wäre entsprechend unserem neutralen Irritationsverständnis ebenso die Gegenrechnung aufzumachen. So haben die Umwälzungen der Berufswelt auch ganz neue Berufsfelder im Medien-, IT- oder Pflegebereich hervorgebracht, die heute Wachstumsbranchen sind; und wer sich darauf eingestellt hatte, hat die guten Chancen für Statusinvestitionen nutzen können. ${ }^{60}$ Ein anderes Beispiel sind die tiefgreifenden Veränderungen von Partnerschaft: Hier sind egalitärere Formen des Umgangs miteinander entstanden, die beiden Seiten neue Optionen der Selbstverwirklichung und Erfahrungen von Anerkennung eröffnet haben.

Doch auch eine Gemengelage negativer und positiver Irritationen kann - selbst wenn Letztere überwögen - eine negative Gesamttönung annehmen, wenn einfach »zu viel auf einmal « disponibel wird, also zu wenige verlässliche Konstanten bleiben. Dann wären Mittelschichtangehörige multiplen Irritationen ausgesetzt; und

57 Koppetsch 2013, S. 121-132, S. 165-177.

58 Greco 2004.

59 Neckel, Wagner 2013.

60 Vogel 2009, S. 215-219.

Leviathan, 42. Jg., 2/2014 
das Multiple dieser Irritationen ergäbe sich nicht nur aus ihrer Vielzahl, sondern darüber hinaus daraus, dass sie erstens zeitlich häufig simultan aufträten und zweitens - wie benannt - vielfache sachliche und soziale Wechselwirkungen zwischen ihnen bestünden. Sie könnten also nicht eine nach der anderen sequentiell abgearbeitet werden, denn sie bildeten nicht bloß eine unverbundene Menge, sondern wären als ein Syndrom zu verstehen.

\subsection{Umgang mit Investitionsstörungen: Praktiken des Coping}

Wie gehen die Mittelschichten mit diesen Irritationen ihrer Lebensführung, die sich in Investitionsstörungen manifestieren, um? Zunächst ist davon auszugehen, dass Irritationen überhaupt nichts Neues sind, sondern gleichsam zur normalen Bewegtheit des Lebens gehören. Solange die Irritationen nicht besonders stark sind und häufiger mal vorkommen, verfügt die Person deshalb über eingespielte Praktiken des Umgangs mit ihnen. Je stärker die Irritationen sind und je kritischere Punkte der Statusarbeit sie betreffen, desto mehr unterliegen Mittelschichtangehörige dann der Erwartung einer möglichst planvollen Bewältigung der Irritationen. Der Planungsimperativ wird gleichsam als »reflexiver Mechanismus «61 auf sich selbst angewandt: Mittelschichtangehörige gehen davon aus, dass planvolle Irritationsbewältigung ein erstrebenswerter und verantwortungsbewusster Modus der Wiederherstellung von Lebensplanung ist und alles andere als illegitim angesehen werden würde.

Mittelschichtangehörige machen sich somit zunächst einmal an eine planvolle Irritationsbewältigung im Sinne einer systematischen Problemsondierung, Alternativensuche und vergleichenden -bewertung, sodass am Ende ein neues nachhaltigeres Arrangement des betreffenden Aspekts der Lebensführung implementiert wird, das die Irritation möglichst vollständig aus der Welt schafft oder deren Wirkungsradius eingrenzt. Wenn jemand zum Beispiel von seinem Arbeitgeber für mehrere Jahre ins Ausland versetzt wird, was beruflich im Falle der Bewährung dort durchaus zu einem Karrieresprung werden kann, aber in einer Familienkonstellation mit einer ebenfalls die eigene Berufskarriere verfolgenden Ehefrau und schulpflichtigen Kindern eine starke Irritation im Sinne einer grundlegenden Störung des mühsam austarierten Arrangements von Beruf, Partnerschaft und Bildungslaufbahn der Kinder darstellt, müsste eine planvolle Bewältigung dieser Irritation entsprechende Informationsbeschaffungen unter anderem über Berufsmöglichkeiten der Frau und schulische Angebote für die Kinder im betreffenden Land, Verhandlungen - etwa mit dem Arbeitgeber der Frau - und gemeinsame Abwägungen und Aushandlungen innerhalb der Familie beinhalten. Wie dieses hier nur angedeutete Beispiel zeigt, wird Irritationsbewältigung durch ein planvolles Neuarrangement der Lebensführung schnell sehr aufwändig, was Informationsverarbeitung, Abstimmung und Konfliktbewältigung anbetrifft - und das unter Bedingungen von manchmal großer Zeitknappheit. Dabei gilt weiterhin, dass mit zunehmender Komplexität der Irritationsbewältigung das Risiko zunimmt, trotz allen Bemühens wichtige Aspekte zu 
übersehen oder falsch einzuschätzen und sich, wie sich dann bald herausstellt, für ein suboptimales oder gar gänzlich verfehltes Neuarrangement zu entscheiden.

Eine durch allgemeine entscheidungssoziologische Überlegungen angeregte Überlegung zur gegenwärtigen Lage der Mittelschichten lautet vor diesem Hintergrund, dass die vermutete Intensivierung und die ebenfalls vermutete Wechselwirkung von Irritationen im Sinne multipler Irritationen dazu führen könnte, dass zunehmend andere, weniger anspruchsvolle Praktiken zum Einsatz gelangen, die wir als Coping bezeichnen. ${ }^{62}$ Coping bleibt weit entfernt von planvollem, Problemen auf den Grund gehendem und sie an der Wurzel packendem Bewältigungshandeln; und Mittelschichtangehörige könnten vor allem deshalb immer häufiger darauf zurückgeworfen werden, weil einem Syndrom wechselwirkender multipler Irritationen eine sprunghafte Komplexitätszunahme innewohnt. Diese wird weiterhin dadurch gefördert, dass Coping Irritationen selten nachhaltig aus der Welt schafft, sondern diese früher oder später in derselben oder anderer Gestalt, und manchmal größer als zuvor, wiederkehren. Es gibt geradezu eine Pfadabhängigkeit des Coping: Wenn heute Irritationen aufgrund ihrer Komplexität nicht nachhaltig bewältigt werden können, erhöht sich dadurch die Wahrscheinlichkeit, dass sie morgen noch komplexer wiederkehren und dann erst recht nicht nachhaltig bewältigt werden können, usw. - was dann zu einer Meta-Irritation darüber werden kann, dass man im eigenen Coping in "planloses Reagieren « verfällt: gleichsam das individuelle Pendant zur schon erwähnten Meta-Irritation über die Politik.

Dies sind freilich Spekulationen, deren empirische Prüfung sehr wichtige Einsichten in die dem Handeln der Mittelschichten zugrunde liegende Logik der Selektion erbringen würde. Zuvor wäre jedoch eine möglichst umfassende Typologie von Coping-Praktiken der Mittelschichten zu konstruieren. Hier können wir erst einmal - ohne Anspruch auf Vollständigkeit - einige Typen anführen, von denen manche möglicherweise weiter in Untertypen aufgliederbar sind. Die Spanne reicht von eher passiven zu aktiven Coping-Praktiken, bei Letzteren von bloßer Identitätsbehauptung zu instrumentellem Coping, und das kann wiederum eher restitutiv oder innovativ ausgerichtet sein. ${ }^{63}$

- Mentale Anpassung: Dieses in manchen Erscheinungsformen nahe am hilflosen Erleiden angesiedelte Coping ist in dem Sinne zunächst passiv, dass nicht sogleich mit irgendeiner Art von Handeln auf Irritationen reagiert wird. Vielmehr werden kognitive, normative oder evaluative Orientierungen angepasst etwa als Verlagerung von Ambitionen von einem Lebensziel auf ein anderes, zum Beispiel von beruflicher Karriere auf ein glückliches Familienleben, als Anspruchsreduktion hinsichtlich Lebenszielen oder Lebenschancen oder als Umdefinieren beispielsweise von beruflichem Stress in eine "sportliche Herausforderung «. Ferner gehört die Identifikation etwa mit den Zumutungen des »unternehmerischen Selbst «64 zur mentalen Anpassung. Im nächsten Schritt

62 Schimank 2011 b.

63 Keine der im Folgenden geschilderten Coping-Praktiken ist als solche mittelschichtspezifisch.

64 Bröckling 2007.

Leviathan, 42. Jg., 2/2014 
ermöglicht diese dann einen neuen handelnden Umgang mit den jeweiligen Irritationen, die fortan nicht mehr als solche erlebt werden.

- »micro-resistance «: $: 55$ Hier wie bei allen im Weiteren genannten Coping-Typen bleibt man, anders als bei der mentalen Anpassung, dauerhaft irritiert und setzt sich niederschwellig aktiv zur Wehr. Dabei geht es bei »micro-resistance « vor allem um Identitätsbehauptung gegenüber irritierenden Handlungszumutungen, also weniger um ein instrumentell auf die Bewältigung der Irritationen ausgerichtetes Handeln. Typische Praktiken sind Verzögerungen und andere Arten von heimlicher Sabotage oder auch »Dienst nach Vorschrift ", den man nicht nur beruflich, sondern auch etwa in der Partnerschaft praktizieren kann. Die Irritationen werden nicht zum Verschwinden gebracht, aber man demonstriert ohne großen Aufwand oder hohe Risiken, dass man die Irritationen als solche empfindet und missbilligt.

- Zeit gewinnen: Diese Art des Coping ist die schwächste, noch eher passive Form des instrumentellen Umgangs mit Irritationen. Man bemüht sich, Irritationen, denen man vorerst wehrlos ausgesetzt ist, auszusitzen, und sorgt so zumindest dafür, dass man weiterhin » am Ball bleibt «. ${ }^{66}$ Dahinter steht die Hoffnung, dass die Irritationen irgendwann auch ohne eigenes Zutun verschwinden oder sich bessere Gelegenheiten für ein aktiveres Coping bieten. ${ }^{67}$

- Mehr Einsatz: Aktiv und instrumentell kann man auf Irritationen reagieren, indem man sich mehr anstrengt und mehr Ressourcen zum Einsatz bringt - in der Hoffnung, so Schwierigkeiten überwinden und die Normalität wiederherstellen zu können. Man kann sich etwa im Beruf angesichts eines Personalabbaus mehr Mühe geben, damit möglichst anderen gekündigt wird, oder angesichts von verlustreichen Finanzmarktinvestitionen jetzt erst recht eine noch größere Summe einsetzen. Wie lange das jeweils durchhaltbar ist, ist allerdings die Frage; denn was man hier zusätzlich investiert, fehlt unter Umständen bald anderswo.

- Externalisierung: Dieser Typ des aktiven und instrumentellen Coping besteht in dem Bemühen, Irritationen auf andere Personen abzuwälzen beziehungsweise anderen Personen die Irritationsbeseitigung aufzuerlegen. So wird vielleicht jemand, der beruflich immer stärker eingespannt ist, mehr und mehr seiner häuslichen oder elterlichen Pflichten der Partnerin zuschieben; und jemand, der größere Verluste bei Finanzmarktgeschäften gemacht hat, wird vielleicht versuchen, seinen Bankberater juristisch haftbar zu machen.

- Kollektiver Protest: Die Artikulation von Forderungen gegenüber denjenigen, die als Verursacher der Irritationen oder als verantwortlich für deren Beseitigung angesehen werden, ist eine weitere aktive und instrumentelle Art des Coping. Letztinstanzlich werden damit vor allem politische Akteure angespro-

65 Dieses Konzept exploriert Anderson (2008) für den Umgang von Wissenschaftlern mit sie irritierenden Hochschulreformen wie etwa dem »Bologna-Prozess « - dies weiterführend siehe Schimank 2014.

66 Schimank 1999.

67 Schimank 2005, S. 404-422. 
chen - mit einer breiten Palette von Aktivitäten, die von Wählerentscheidungen über weitere konventionelle bis hin zu unkonventionellen Formen politischer Partizipation zum Beispiel nach Art der Stuttgarter »Wutbürger « reichen.

- »Exit«: Wenn man die Lage einerseits so einschätzt, dass man Irritationen nicht selbst bewältigen kann, und andererseits auch keine Chancen für erfolgversprechenden kollektiven Protest sieht, kann der - nicht bloß mentale oder, wie bei "micro-resistance ", symbolische - Rückzug aus den jeweiligen Störfeldern eine noch verbleibende Möglichkeit des Coping sein. Das Spektrum umfasst hier Praktiken wie Kündigung, Scheidung, den Wechsel von Finanzmarktgeschäften zu anderen Formen der Geldanlage wie Immobilienkauf - und am extremsten Auswanderung in ein anderes Land. "Exit " ist aktiv und instrumentell und von der Hoffnung getragen, dass anderswo die jeweiligen Irritationen nicht oder in geringerem Maße existieren.

- Improvisation: ${ }^{68}$ Die bis jetzt geschilderten Typen des aktiven instrumentellen Coping sind überwiegend restitutiv in dem Sinne, dass sie möglichst die ursprünglichen Verhältnisse wiederherstellen wollen. Improvisation ist ebenfalls aktiv und instrumentell, aber dabei innovativ ausgerichtet, sucht also mit bescheidenem Aufwand ad hoc nach neuen Wegen der Irritationsbewältigung diesbezüglich analog zum planvollen Neuarrangement der Lebensführung. Hier geht es um »Basteln, Flicken, Probieren, Kombinieren « ${ }^{69}$, wobei man zumeist, um schnell auf Irritationen reagieren zu können, ohne größeres Nachdenken auf situativ bereitstehende Ressourcen und gegebenenfalls mobilisierbare andere zurückgreift. Wenn zum Beispiel das eigene Kind auf dem Gymnasium nicht mitkommt, was eine starke Irritation der intergenerationalen Statusinvestitionen ist, und man von einem Arbeitskollegen berichtet bekommt, dass er gerade sein Kind bei einer neu etablierten Waldorfschule angemeldet hat, tut man ohne viel zu überlegen dasselbe und wartet dann ab, wie sich die Dinge weiter entwickeln.

Wie hier und da angedeutet, gibt es vielfältige Verknüpfungen dieser Coping-Typen, denen systematisch nachzugehen wäre. $\mathrm{Zu}$ jedem Coping-Typ existieren bereits Forschungen - freilich kaum spezifisch auf Mittelschichtangehörige und deren verschiedene Investitionsstörungen bezogen. Völlig fehlen dürften jedoch Coping-Profile von Mittelschichtangehörigen, also empirisch fundierte Aufarbeitungen des Ensembles von einzeln oder kombiniert eingesetzten Coping-Praktiken über die verschiedenen Störfelder der Lebensführung hinweg. Genau solche Profile müssten aber, zur Ergänzung der Investitionskalküle, ein zentraler Bestandteil der soziologischen Charakterisierung der Mittelschichten sein.

68 Hierzu, am Beispiel der Bewohner einer südamerikanischen Kleinstadt, sehr plastisch Guttandin 1996.

69 Ebd., S. 31. 


\subsection{Aggregationsdynamiken: Mittelschichtgetriebener gesellschaftlicher Wandel}

Bis hierher ist der primäre Bezugspunkt der Fragerichtungen, die das erläuterte Forschungsprogramm beinhaltet, der je einzelne Mittelschichtangehörige beziehungsweise -haushalt. Wenn man nun den Bezugspunkt hin zur Frage der gesellschaftlichen Ordnung wechselt, dann stehen die Effekte des massenhaften Zusammenwirkens von Mittelschichtangehörigen und anderen gesellschaftlichen Akteuren auf gesellschaftsstrukturelle Dynamiken im Vordergrund. Um nur ein Beispiel zu geben: Heinz Bude spekuliert darüber, dass die »Bildungspanik « vieler Mittelschichteltern in Bezug auf ihre Kinder zu einer ruinösen Konkurrenz nach Art eines Rüstungswettlaufs führen könnte. ${ }^{70}$ Viele Eltern rüsten demzufolge ihre Kinder in Sachen schulischer Leistungsanforderungen durch alle möglichen Arten zusätzlicher Unterstützungen mehr und mehr auf - mit dem Ergebnis, dass kein Kind dadurch einen entscheidenden Vorsprung erringen kann, aber alle völlig » bildungsüberanstrengt « werden - ganz zu schweigen von den durch derart fordernde Eltern und geforderte Kinder ebenfalls überforderten Lehrern.

Die Bearbeitung solcher Fragen muss zwei Arten von Handeln und Handlungseffekten auseinanderhalten, die beide stattfinden und miteinander in Wechselwirkung stehen. Zum einen gibt es Aktivitäten, mit denen Mittelschichtangehörige gemeinsam versuchen, die Bedingungen ihrer Lebensführung, insbesondere ihrer Statusarbeit, möglichst vorteilhaft zu gestalten, vor allem als politisches Engagement etwa in Form von kollektivem Protest. Diese intentionalen, oft auf die Veränderung der Baupläne von Institutionen zielenden Bemühungen der Gesellschaftsgestaltung sind das kollektive Pendant zum planvollen Neuarrangement der je individuellen Lebensgestaltung, mögen sie glücken oder an der Komplexität der Verhältnisse scheitern. Zum anderen, und mitunter weit wirkungsmächtiger, stellen sich aus dem Bewältigungshandeln, insbesondere den Coping-Praktiken der verschiedenen Mittelschicht-Fraktionen, transintentionale gesamtgesellschaftliche Aggregationseffekte ein. Es wäre wichtig, die Aufmerksamkeit soziologischer Gesellschaftsbeobachtung besonders auf diese Effekte zu lenken, weil sie oft als unangenehme Überraschungen erlebt werden. Doch hier warten erst einmal viele ungelöste theoretische und empirische Probleme. ${ }^{71}$ Es ist weitgehend ungeklärt, wie sich derartige Aggregationsdynamiken theoretisch konzeptualisieren und empirisch untersuchen lassen.

Wir gehen davon aus, dass die Coping-Praktiken der Mittelschichten drei Arten ineinandergreifender Aggregationseffekte freisetzen: erstens Aufsummierungen unverbundenen individuellen Copings, zweitens horizontale Interdependenzen individuellen Copings, also übergreifende Effekte, die sich aus der Coping-Praxis mehrerer Individuen ergeben, und drittens vertikale Interdependenzen von individuellem Coping und dem Agieren von Organisationen in den verschiedenen Gesellschaftsbereichen.

70 Bude 2011.

71 Greshoff et al. 2011. 
Individuelle Coping-Praktiken summieren sich zu Struktureffekten wie etwa Scheidungsraten, dem Wachstum von Investmentfonds oder dem Anstieg beruflicher Mobilität auf. Bei diesen Aufsummierungen massenhaften, nicht selten gleichartigen Handelns aufgrund gleichartiger Situationsdeutungen ${ }^{72}$ muss es zwischen den Handelnden keine soziale Beziehung im Sinne einer sinnhaften Orientierung aneinander geben. Die Struktureffekte prägen dann weiteres Handeln, ob die Akteure sie registrieren oder nicht. Scheidungsraten beispielsweise sind einer der Bestimmungsfaktoren der Chancen auf Heiratsmärkten. Wenn Mittelschichtangehörige wissen, dass die Raten steigen, kann ihnen das die eigene Entscheidung zur Scheidung erleichtern: Denn der Markt ist ja in Bewegung. Aber selbst wenn sie sich in Unwissenheit über die Scheidungsraten zur Scheidung entschließen, sind ihre Chancen, einen neuen Partner zu finden, bei hohen größer als bei geringen Raten.

Zweitens können individuelle Coping-Praktiken auch »Schachzüge « in horizontalen Interdependenzen mit dem Coping anderer Individuen sein. Dazu genügt wechselseitige Beobachtung; wenn Möglichkeiten wechselseitiger Beeinflussung hinzukommen, kann Coping aktiver in dem Sinne werden, dass man sich nicht mehr nur dem tatsächlichen oder antizipierten Handeln der vielen anderen anpasst, sondern versucht, deren Handeln durch Versprechungen und Drohungen so zu beeinflussen, dass es dem, was man selbst tun will, möglichst zuträglich ist. ${ }^{73}$ Wechselseitige Beobachtung und Beeinflussung bringen durch das Reagieren aufeinander bestimmte Eigendynamiken in Gang, etwa Diffusions- und Eskalationsprozesse, in denen es zu Nachahmungen, Kooperationen, wechselseitigen Anregungen, Lernen voneinander, Vertrauensspiralen, Konkurrenzintensivierungen, sozialen Schließungen, Überbietungen, » Matthäus-Effekten « ${ }^{74}$ oder sich selbst erfüllenden Prophezeiungen kommen kann. ${ }^{75}$

Drittens müssen dann auch noch die vertikalen Interdependenzen von Mittelschichtangehörigen und denjenigen Organisationen, mit denen sie in den verschiedenen Gesellschaftsbereichen zu tun haben, mit in den Blick genommen werden also etwa von Eltern und Schulen, Beschäftigten und Arbeitgebern, politisch Engagierten und Parteien, Kleinanlegern und Banken oder Versicherten und Versicherungsträgern. Das Leistungsangebot der Organisationen und dessen Wandel können Möglichkeitsspielräume der Individuen eröffnen oder verschließen; und die Individuen können versuchen, auf die Ausgestaltung des Leistungsangebots Einfluss zu nehmen.

Erst aus dem Wechselspiel von horizontalem und vertikalem handelnden Zusammenwirken sind viele Aggregationsdynamiken zu begreifen, die sich in gesellschaftlichen Struktureffekten niederschlagen, wie sie sich etwa in Gestalt von Arbeitsmarktchancen, Einkommensverteilungen, Wertewandel, Leitbildern elterlichen

72 Weber 1972 [1922], S. 11-12.

73 Schimank 2000, S. 247-259.

74 Merton 1985 [1968]; DiPrete, Eirich 2006; Diewald, Faist 2011.

75 Zur Modellierung solcher Dynamiken verfügen die Sozialwissenschaften über eine ganze Reihe theoretischer Mechanismen - als Sammlungen siehe Elster (2007, S. 299-443) sowie Neckel et al. (2010). 
oder partnerschaftlichen Handelns oder Rationalitätsmythen des Geldanlegens und ganz übergreifend als jeweilige Position der Mittelschichten in der gesamtgesellschaftlichen Kräftekonstellation - als Lebensumstände und Lebenschancen der je einzelnen Mittelschichtangehörigen manifestieren. Diese Struktureffekte können dann wiederum für alle Beteiligten mehr oder weniger relevant werden. Sie können insbesondere erneut irritierend wirken und dadurch weiteres Bewältigungshandeln hervorrufen.

Ein auch in seiner Komplexität anschauliches Beispiel für das Zusammenspiel horizontaler und vertikaler Wechselwirkungen stellt der Finanzmarkt dar. Hier interagieren Mittelschichtangehörige mit ihren Investitionsstrategien mit Millionen anderer Kleinanleger - die meisten davon aus den Mittelschichten vieler anderer Länder - sowie mit einer ganzen Reihe von Organisationen, die ihrerseits vielfältig untereinander interagieren: vor allem Banken, Fonds, Rating-Agenturen, nationale und internationale Zentralbanken sowie nationale und supranationale Regierungen. ${ }^{76}$ Gerade weil die Kleinanleger auch insgesamt keine entscheidenden Teilnehmer am Börsenspiel sind (und noch weniger jeder Einzelne von ihnen), kann man das, was ihnen widerfährt und immer wieder zu tiefer Hilflosigkeit führt, ${ }^{77}$ nur aus den Dynamiken dieser Gesamtkonstellation begreifen. Die analytischen Herausforderungen solcher Mehrebenenkonstellationen sind allerdings sehr groß und wurden, abgesehen von einzelnen Fallstudien, bislang noch kaum angegangen; generalisierbare theoretische Mechanismen fehlen fast völlig. ${ }^{78}$ Doch ein angemessenes Verständnis dessen, was heute die Mittelschichten bewegt, muss diesen komplizierten Wegen nachgehen, auf denen je individuelle Lebensführungen kollektive Lebensumstände hervorbringen, die als je individuelle Lebenschancen wiederum die weiteren Lebensführungen prägen.

\section{Schlussbetrachtung}

Im vorliegenden Beitrag haben wir zwei Ziele verfolgt. Zum einen haben wir ein theoretisches Modell der Lebensführung von Mittelschichtangehörigen als Statusarbeit vorgelegt, zum anderen mit diesem Modell eine mögliche theoretische Herangehensweise an zeitdiagnostische Fragen zur Lage der Mittelschichten in Deutschland heute skizziert. Dass es sich um Skizzen handelt, bedeutet, dass wir bisher noch keine empirische Prüfung unserer theoretischen Überlegungen vornehmen konnten. Unser Anliegen bestand darin, weiterer empirischer Forschung eine theoretische Richtungsweisung zu geben, an der es derzeit mangelt. Theoretische Vorschläge können keine empirischen Fragen beantworten und damit auch in der gesellschaftspolitischen Debatte darüber, was bezüglich der Mittelschichten getan werden sollte, keine Stellung beziehen. Wir gehen allerdings davon aus, dass die von uns vorge-

76 Mau 2014.

77 Schimank 2011c.

78 Mayntz 2002. 
schlagene theoretische Orientierung der empirischen Forschung deren sozialwissenschaftliche und gesellschaftspolitische Relevanz nachhaltig steigern kann.

Am Ende derart theoretischer Analysen ließe sich vermutlich dann auch die Frage beantworten, wie es um die künftige sozialstrukturelle wie kulturelle Hegemonie der Mittelschichten steht. Göran Therborn hat, an Perspektivpapiere der OECD anknüpfend, gefragt, ob wir im globalen Maßstab vielleicht gar » a coming middleclass century " heraufkommen sehen. ${ }^{79}$ Wie entwickelt sich die gesellschaftliche Positionierung der Mittelschichten im longitudinalen und internationalen Vergleich? Werden sie weiterhin hierzulande und im »alten « Europa und zunehmend auch in anderen sich entwickelnden Weltregionen wie etwa den BRICS-Ländern nicht nur die größte Bevölkerungsgruppe sein beziehungsweise werden, sondern auch mit ihrer Art der Lebensführung die »gute" Gesellschaft repräsentieren? Und wie muss sich diese Lebensführung veränderten und sich weiter verändernden gesellschaftlichen Bedingungen anpassen? Anderswo auf der Welt, etwa in Südostasien, beobachten wir im Gefolge wirtschaftlicher Wachstumsprozesse ein Wachstum der Mittelschichten, das bislang vor allem im Konsum seinen Ausdruck findet, während erst langsam politische und soziale Ansprüche formuliert werden. ${ }^{80}$ In wieder anderen Ländern, so beispielsweise in Nordafrika, sind politisch wie ökonomisch enttäuschte Mittelschichten an entscheidenden innergesellschaftlichen Auseinandersetzungen, vor allem mit Fundamentalisten, beteiligt und versuchen sich gesellschaftlich zu behaupten.

Diese divergierenden Entwicklungen werfen lauter derzeit unbeantwortbare Fragen auf. Klar ist allerdings: Wer heute die Zukunftsfähigkeit nicht nur unserer westlichen Gesellschaften abschätzen will und vielleicht auch Hinweise darauf geben möchte, wie diese Zukunftsfähigkeit gesteigert oder überhaupt erst einmal wiederhergestellt werden kann, wird nicht darum herumkommen, sich ganz prominent mit den Mittelschichten zu beschäftigen. Wo sie in den nächsten Jahren ihr Gewicht in die Waagschale werfen werden, ist zwar sicher nicht die einzige, aber doch eine der wichtigen Determinanten der mittel- und langfristigen globalen Entwicklung. Dabei wird entscheidend sein, auf welche Weise die Mittelschichten mit Irritationen ihrer Lebensführung umgehen - insbesondere inwieweit sie im Spektrum von Reaktanz und Statusverteidigung einerseits und Innovation und Selbsttransformation andererseits in der Lage sind, ein zukunftsträchtiges gesellschaftliches Projekt zu definieren und letztlich auch durchzusetzen.

\section{Literatur}

Abbott, Andrew 1988. The system of professions. An essay on the division of expert labor. Chicago: University of Chicago Press.

Allum, Percy 1995. State and society in Western Europe. Cambridge: Polity Press.

Anderson, Gina 2008. "Mapping academic resistance in the managerial university «, in Organization 15, S. 251-270.

79 Therborn 2012, S. 15-17.

80 Lange, Meier 2009.

Leviathan, 42. Jg., 2/2014 
Arthur, Brian 1989. »Competing technologies. Increasing returns, and lock-in by historical events ", in The Economic Journal 99, S. 116-131.

Bagnasco, Arnaldo. Hrsg. 2008. Ceto medio. Perché e come occuparsene. Una ricerca del Consiglio italiano per le Scienze Sociali. Bologna: Il Mulino.

Beck, Ulrich 1986. Die Risikogesellschaft. Auf dem Weg in eine andere Moderne. Frankfurt a. M.: Suhrkamp.

Berger, Johannes 1996. "Was behauptet die Modernisierungstheorie wirklich - und was wird ihr nur unterstellt? ", in Leviathan 24, S. 45-62.

Boltanski, Luc; Chiapello, Ève 2003. Der neue Geist des Kapitalismus. Konstanz: UVK.

Bolte, Karl M. 1966. Deutsche Gesellschaft im Wandel. Band 1 (mit Beiträgen von Dieter Kappe, Katrin Aschenbrenner, Friedhelm Neidhardt). Opladen: Leske.

Bourdieu, Pierre 1979. Die feinen Unterschiede. Zur Kritik der gesellschaftlichen Urteilskraft. Frankfurt a. M.: Suhrkamp.

Bourdieu, Pierre 1986. »Die biographische Illusion «, in BIOS 3, S. 75-81.

Bröckling, Ulrich 2007. Das unternehmerische Selbst. Soziologie einer Subjektivierungsform. Frankfurt a. M.: Suhrkamp.

Bude, Heinz 2011. Bildungspanik. Was unsere Gesellschaft spaltet. München: Hanser.

Buhr, Petra; Feldhaus, Michael. Hrsg. 2012. Die notwendige Vielfalt von Familie und Partnerschaft. Würzburg: Ergon.

Burkhardt, Christoph et al. 2012. Mittelschicht unter Druck? Gütersloh: Verlag Bertelsmann Stiftung.

Castel, Robert; Dörre, Klaus. Hrsg. 2009. Prekarität, Abstieg, Ausgrenzung. Die soziale Frage am Beginn des 21. Jahrhunderts. Frankfurt a. M: Campus.

Chauvel, Luis 2006. Les classes moyennes à la dérive. Paris: Le Seuil.

Collado, Emanuel 2010. The shrinking middle class. Why America is becoming a two-class society. Bloomington: iUniverse.

Dahrendorf, Ralf 1992. Der moderne soziale Konflikt. Essay zur Politik der Freiheit. Stuttgart: Deutsche Verlagsanstalt.

Deutschmann, Christoph 2005. "Finanzmarkt-Kapitalismus und Wachstumskrise», in Finanzmarkt-Kapitalismus. Analysen zum Wandel von Produktionsregimen. Kölner Zeitschrift für Soziologie und Sozialpsychologie, Sonderheft 45, hrsg. v. Windolf, Paul, S. 58-84. Wiesbaden: VS Verlag für Sozialwissenschaften.

DiPrete, Thomas A.; Eirich, Gregory M. 2006. "Cumulative advantage as a mechanism for inequality. A review of theoretical and empirical developments ", in Annual Review of Sociology 32, S. 271-297.

Diewald, Martin; Faist, Thomas 2011. "Von Heterogenitäten zu Ungleichheiten: Soziale Mechanismen als Erklärungsansatz der Genese sozialer Ungleichheiten ", in Berliner Journal für Soziologie 21, S. 91-114.

Doering-Manteuffel, Anselm; Raphael, Lutz 2008. Nach dem Boom. Perspektiven auf die Zeitgeschichte seit 1970. Göttingen: Vandenhoeck \& Ruprecht.

Dürrschmidt, Jörg 2002. Globalisierung. Bielefeld: transcript.

Ehrenreich, Barbara 1989. Fear of falling. The inner life of the middle class. New York: Pantheon.

Elder, Glen 1998. »The life course as developmental theory «, in Child Development 69, S. 1-12.

Elster, Jon 2007. Explaining social behavior. More nuts and bolts for the social sciences. Cambridge: Cambridge University Press.

Erikson, Robert; Goldthorpe, John H. 1992. The constant flux: a study of class mobility in industrial societies. Oxford: Clarendon Press.

Esser, Hartmut 1993. Soziologie - Allgemeine Grundlagen. Frankfurt a. M.: Campus.

Esser, Hartmut 2000. Soziologie - Spezielle Grundlagen. Band 4: Opportunitäten und Restriktionen. Frankfurt a. M.: Campus.

Fourquet, Jerome; Mergier, Alain; Peugny, Camille 2013. Le grand malaise. Enquete sur les classes moyennes. Paris: Jean Jaures Fondation.

Gornick, Janet C.; Jäntti, Markus 2013. Income inequality: economic disparities and the middle class in affluent countries. Palo Alto: Stanford University Press. 
Greco, Monica 2004. »Wellness «, in Glossar der Gegenwart, hrsg. v. Bröckling, Ulrich; Krasmann, Susanne; Lemke, Thomas, S. 293-299. Frankfurt a. M..: Suhrkamp.

Greshoff, Rainer; Huinink, Johannes; Schimank, Uwe 2011. Aggregation dynamics of multi-level actor constellations: introductory paper. Welfare Societies Working Paper Nr. 02. Universität Bremen: Forschungsverbund »Welfare Societies«.

Groh-Samberg, Olaf 2002. »Neoliberalismus als hegemoniales Projekt. Zur Erklärungskraft der politischen Soziologie Pierre Bourdieus ", in Theorie als Kampf? Zur politischen Soziologie Pierre Bourdieus, hrsg. v. Bittlingmayer, Uwe et al., S. 197-224. Opladen: Leske + Budrich.

Groh-Samberg, Olaf 2009. »Sorgenfreier Reichtum: Jenseits von Konjunktur und Krise lebt nur ein Prozent der Bevölkerung", in DIW Wochenbericht 76, 35, S. 590-597.

Guttandin, Friedhelm 1996. Improvisationsgesellschaft. Provinzstadtkultur in Südamerika. Pfaffenweiler: Centaurus.

Hacker, Jacob S. 2006. The great risk shift: the assault on American jobs, families, health care and retirement - and how you can fight back. Oxford: Oxford University Press.

Hacker, Jacob; Pierson, Paul 2011. Winner-take-all politics. How Washington made the rich richer - and turned its back on the middle class. New York: Simon \& Schuster.

Heinze, Rolf 2011. Die erschöpfte Mitte. Zwischen marktbestimmten Soziallagen, politischer Stagnation und der Chance auf Gestaltung. Weinheim: Juventa.

Held, David. Hrsg. 2000. A globalizing world? Culture, economics, politics. London: Routledge.

Herbert-Quandt-Stiftung. Hrsg. 2007. Zwischen Erosion und Erneuerung. Die gesellschaftliche Mitte in Deutschland. Frankfurt a. M.: Societäts-Verlag.

Herrmann, Ulrike 2010. Hurra, wir dürfen zablen. Der Selbstbetrug der Mittelschicht. Frankfurt a. M.: Westend.

Hirschman, Albert O. 1970. Exit, voice and loyalty: responses to decline in firms, organizations, and states. Cambridge: Harvard University Press.

Hradil, Stefan; Schmidt, Holger 2007. "Angst und Chancen. Zur Lage der gesellschaftlichen Mitte aus soziologischer Sicht ", in Zwischen Erosion und Erneuerung. Die gesellschaftliche Mitte in Deutschland. Ein Lagebericht, hrsg. v. Herbert-Quandt-Stiftung, S. 163-234. Frankfurt a. M.: Societäts-Verlag.

Illouz, Eva 2007. Cold intimacies. The making of emotional capitalism. Cambridge: Polity Press.

Jarausch, Konrad H. Hrsg. 2008. Das Ende der Zuversicht? Die siebziger Jahre als Geschichte. Göttingen: Vandenhoeck \& Ruprecht.

Jungbauer-Gans, Monika; Lohmann, Henning; Spiess, Katharina C. 2012. »Bildungsungleichheiten und Privatschulen in Deutschland ", in Soziologische Bildungsforschung, hrsg. v. Solga, Heike; Becker, Rolf, S. 64-85. Wiesbaden: Springer VS.

Knötig, Nora 2010. »Schließungsprozesse innerhalb der bildungsbürgerlichen Mitte", in Dynamiken (in) der gesellschaftlichen Mitte, hrsg. v. Burzan, Nicole; Berger, Peter A., S. 331-354. Wiesbaden: VS Verlag für Sozialwissenschaften.

Koppetsch, Cornelia 2013. Die Wiederkehr der Konformität. Streifzüge durch die gefährdete Mitte. Frankfurt a. M.: Campus.

Lange, Hellmuth; Meier, Lars. Hrsg. 2009. Globalizing lifestyles, consumerism and environmental concern - the case of the new middle classes. Berlin, New York: Springer.

Lerner, Daniel 1958. The passing of traditional society: modernizing the Middle East. London: Macmillan.

Lessenich, Stephan 2008. Die Neuerfindung des Sozialen. Der Sozialstaat im flexiblen Kapitalismus. Bielefeld: transcript.

Luhmann, Niklas 1974 [1966]. »Reflexive Mechanismen«, in Niklas Lubmann: Soziologische Aufklärung. Band 1: Aufsätze zur Theorie sozialer Systeme, S. 92-112. Opladen: Westdeutscher Verlag.

Marg, Stine et al. Hrsg. 2013. Die neue Macht der Bürger. Was motiviert die Protestbewegungen? BP-Gesellschaftsstudie. Reinbek bei Hamburg: Rowohlt.

Mau, Steffen 2012. Lebenschancen. Wohin driftet die Mittelschicht? Berlin: Suhrkamp.

Mau, Steffen 2013. "European middle classes in trouble? ", in Perspectives on Europe 43, 1, S. 27-31. 
Mau, Steffen 2014. »Transformation und Krise der europäischen Mittelschichten «, in Krise der europäischen Vergesellschaftung? Soziologische Perspektiven, hrsg. v. Heidenreich, Martin. Wiesbaden: Springer VS (im Erscheinen).

Mayntz, Renate 2002. "Zur Theoriefähigkeit makro-sozialer Analysen «, in Akteure - Mechanismen - Modelle. Zur Theoriefähigkeit makro-sozialer Analysen, hrsg. v. Mayntz, Renate, S. 7-43. Frankfurt a. M.: Campus.

Merton, Robert K. 1985 [1968]. »Der Matthäus-Effekt in der Wissenschaft «, in Robert K. Merton: Entwicklung und Wandel von Forschungsinteressen, S. 100-116. Frankfurt a. M.: Suhrkamp.

Mudge, Stephanie Lee 2008. "What is neo-liberalism?", in Socio-Economic Revue 6, 4, S. 703-731.

Müller, Hans-Peter 1992. Sozialstruktur und Lebensstile: die neuere theoretische Diskussion über soziale Ungleichheit. Frankfurt a. M.: Suhrkamp.

Münch, Richard 1986. Die Kultur der Moderne. 2 Bände. Frankfurt a. M.: Suhrkamp.

Münkler, Herfried 2010. Mitte und Maß. Der Kampf um die richtige Ordnung. Berlin: Rowohlt.

Neckel, Sighard 2008. Flucht nach vorn. Die Erfolgskultur der Marktgesellschaft. Frankfurt a. M., New York: Campus.

Neckel, Sighard et al. Hrsg. 2010. Sternstunden der Soziologie. Wegweisende Theoriemodelle des soziologischen Denkens. Frankfurt a. M.: Campus.

Neckel, Sighard; Wagner, Greta. Hrsg. 2013. Leistung und Erschöpfung. Bournout in der Wettbewerbsgesellschaft. Berlin: edition suhrkamp.

Newman, Katherine 2012. The accordion family. Boomerang kids, anxious parents and the private toll of global competition. Boston: Beacon Press.

Nolte, Paul; Hilpert, Dagmar 2007. "Wandel und Selbstbehauptung. Die gesellschaftliche Mitte in historischer Perspektive", in Zwischen Erosion und Erneuerung. Die gesellschaftliche Mitte in Deutschland. Ein Lagebericht, hrsg. v. Herbert-Quandt-Stiftung, S. 11-101. Frankfurt a. M.: Societäts-Verlag.

Picketty, Thomas 2014. Capital in the twenty-first century. Cambridge: Belknap Press.

Rehbein, Boike; Saalmann, Gernot 2009 a. »Habitus «, in Bourdieu Handbuch, hrsg. v. Fröhlich, Gerhard; Rehbein, Boike, S. 110-118. Stuttgart: Metzler.

Rehbein, Boike; Saalmann, Gernot 2009 b. »Kapital « in Bourdieu Handbuch, hrsg. v. Fröhlich, Gerhard; Rehbein, Boike, S. 110-118. Stuttgart: Metzler.

Rosa, Hartmut 2006. Beschleunigung. Die Veränderung der Zeitstrukturen der Moderne. Frankfurt a. M.: Suhrkamp.

Rossi, Alice S.; Rossi, Peter H. 1990. Of human bonding: parent-child relations across the life course. New York: Aldine.

Rössel, Jörg; Otte, Gunnar. Hrsg. 2011. Lebensstilforschung. Kölner Zeitschrift für Soziologie und Sozialpsychologie, Sonderheft 51. Wiesbaden: VS Verlag für Sozialwissenschaften.

Schäfer, Armin 2013. "Liberalization, inequality and democracy's discontent ", in Politics in the age of austerity, hrsg. v. Schäfer, Armin; Streeck, Wolfgang, S. 169-195. Cambridge: Polity Press.

Schelsky, Helmut 1953. Wandlungen der deutschen Familie in der Gegenwart. Stuttgart: Enke.

Schimank, Uwe 1999. »Flipperspielen und Lebenskunst ", in Identität und Moderne, hrsg. v. Willems, Herbert; Hahn, Alois, S. 250-272. Frankfurt a. M.: Suhrkamp.

Schimank, Uwe 2000. Handeln und Strukturen. Einführung in die akteurtheoretische Soziologie. Weinheim: Juventa.

Schimank, Uwe 2005. Die Entscheidungsgesellschaft. Komplexität und Rationalität der Moderne. Wiesbaden: VS Verlag für Sozialwissenschaften.

Schimank, Uwe 2011 a. "Against all odds. The >loyalty` of small investors ", in Socio-Economic Review 9, S. 107-135.

Schimank, Uwe 2011 b. »Nur noch Coping. Eine Skizze postheroischer Politik «, in Zeitschrift für Politikwissenschaft 21, 3, S. 455-463.

Schimank, Uwe 2011 c. »Die >Hyperkomplexität des Finanzmarkts und die Hilflosigkeit der Kleinanleger «, in Leviathan 39, S. 499-517. 
Schimank, Uwe 2014. »Identitätsbedrohungen und Identitätsbehauptung: Professoren in reformbewegten Universitäten ", in Formalität und Informalität in Organisationen, hrsg. v. Groddeck, Victoria von; Wilz, Sylvia. Wiesbaden: Springer VS (im Erscheinen).

Schimank, Uwe; Stopper, Silke 2012. »Kleinanleger auf dem Finanzmarkt: Praktiken der Hilflosigkeitsabsorption", in Entfesselte Finanzmärkte - Soziologische Analysen des modernen Kapitalismus, hrsg. v. Kraemer, Klaus; Nessel, Sebastian, S. 243-261. Frankfurt a. M.: Campus.

Schulze, Gerhard 1992. Die Erlebnis-Gesellschaft. Kultursoziologie der Gegenwart. Frankfurt a. M.: Campus.

Solga, Heike; Wimbauer, Christine. Hrsg. 2005. "Wenn zwei das Gleiche tun ... ". Ideal und Realität sozialer (Un-)Gleichheit in Dual Career Couples. Opladen: Barbara Budrich.

Streeck, Wolfgang 2013. Gekaufte Zeit. Die vertagte Krise des demokratischen Kapitalismus. Berlin: Suhrkamp.

Therborn, Göran 2012. »Class in the 21st century ", in New Left Review 78, S. 5-29.

Vester, Michael et al. 2001. Soziale Milieus im gesellschaftlichen Strukturwandel. Zwischen Integration und Ausgrenzung. Frankfurt a. M.: Suhrkamp.

Vogel, Berthold 2009. Wohlstandskonflikte. Soziale Fragen, die aus der Mitte kommen. Hamburg: Hamburger Edition.

Vogel, Berthold 2011. »Mittelschicht zwischen Abstiegsängsten und hoher Belastung «, in Wirtschaftsdienst 8, S. 507-510.

Voß, Gerd-Günter 1991. Lebensführung als Arbeit. Über die Autonomie der Person im Alltag der Gesellschaft. Stuttgart: Enke.

Voß, Gerd-Günter; Pongratz, Hans J. 1998. »Der Arbeitskraftunternehmer. Eine neue Grundform der Ware Arbeitskraft? «, in Kölner Zeitschrift für Soziologie und Sozialpsychologie 50, 1, S. 131-158.

Weber, Max 1975 [1905]. »Die protestantische Ethik und der Geist des Kapitalismus «, in Max Weber: Die protestantische Ethik I, S. 27-277. Hamburg: Siebenstern.

Weber, Max 1963 [1919]. Gesammelte Aufsätze zur Religionssoziologie I. Tübingen: Mohr.

Weber, Max 1972 [1922]. Wirtschaft und Gesellschaft. 5., rev. Auflage. Tübingen: Mohr.

Wimbauer, Christine 2012. Wenn Arbeit Liebe ersetzt. Doppelkarriere-Paare zwischen Anerkennung und Ungleichheit. Frankfurt a. M., New York: Campus.

Windolf, Paul. Hrsg. 2005. Finanzmarkt-Kapitalismus. Analysen zum Wandel von Produktionsregimen. Kölner Zeitschrift für Soziologie und Sozialpsychologie, Sonderheft 45. Wiesbaden: VS Verlag für Sozialwissenschaften. 
Zusammenfassung: Die Debatten über die Verunsicherungen oder gar Krisen der Mittelschichten der letzten Jahre erweisen sich zumeist als nicht hinreichend theoretisch fundiert und beruhen oft auf einem oberflächlichen beziehungsweise bruchstückhaften Verständnis dessen, was die Mittelschichten ausmacht. Um zu einer genaueren Klärung der generellen Logik der Lebensführung der Mittelschichten zu gelangen, schlagen wir im ersten Teil dieses Beitrags eine Antwort vor, die diese Logik so, wie sie in der sozialstrukturellen Verortung der Mittelschichten angelegt ist, auf eine neue Weise als Investieren in den Status theoretisch ausbuchstabiert. Im zweiten Teil zeigen wir, wie auf dieser analytischen Grundlage zeitdiagnostische Fragen angesprochen werden könnten. Welche Zusammenhänge bestehen zwischen heutigen gesellschaftlichen Veränderungsdynamiken auf der einen und Veränderungen der Lebensumstände und Lebensführung der Mittelschichten auf der anderen Seite? Hierzu stellen wir ein systematisch angelegtes Forschungsprogramm mit Leitfragen und Schlüsselkonzepten vor.

Stichworte: Mittelschichten, Statusarbeit, Irritationen, Coping

\title{
Investing in status: the middle classes' demanding mode of living
}

Summary: Recently there have been debates about the gropwing feelings of insecurity or even the crisis among the middle classes. But these debates have no substantial theoretical foundation and are often based on a superficial and incomplete understanding of the middle classes. To reach a more precise clarification of the general logic of the middle classes' conduct of life, the first part of this article proposes a new answer, which spells out this logic, which is based in the social structural position of the middle classes, as an ongoing investment in status. The second part shows how this analytical model can be used to address questions about the current situation of the middle classes. How are the ongoing dynamics of societal change, on the one hand, related to the circumstances of life and mode of living of the middle classes, on the other? To prepare the way for further investigations, a systematic research agenda with key questions and key concepts has been developed.

Keywords: middle classes, status work, irritations, coping

\author{
Autoren \\ Prof. Dr. Olaf Groh-Samberg \\ Prof. Dr. Steffen Mau \\ Prof. Dr. Uwe Schimank \\ Alle: \\ Institut für Soziologie \\ Fachbereich 08 / Sozialwissenschaften \\ Universität Bremen \\ UNICOM \\ Mary-Somerville-Str. 9 \\ 28359 Bremen
}

